\title{
STRUCTURAL PROPERTIES OF SPARSE GRAPHS
}

\author{
JAROSLAV NEŠETŘIL AND PATRICE OSSONA DE MENDEZ
}

\section{Contents}

1. Introduction 2

1.1. Dense Graphs 2

1.2. Sparse Graphs 3

1.3. Nowhere Dense Graphs 5

2. Measuring sparsity 5

2.1. Shallow minors and Grads 6

2.2. Shallow Topological Minors and Top-grads 7

2.3. Hajós or Hadwiger? 9

2.4. Stability with Respect to Lexicographic Product 9

3. Sparse Classes of Graphs 11

3.1. Basic Definitions 11

3.2. When is a Class Sparse or Dense? 12

3.3. Within the Nowhere Dense World 14

3.4. Classes with Bounded Expansion 16

3.5. Proper Minor Closed Classes 18

3.6. The Full Picture 19

4. Regular Partitions of Sparse Graphs 21

4.1. Tree-width 21

4.2. Tree-depth 21

4.3. Generalized Coloring Numbers 24

4.4. Low tree-width coloring 26

4.5. Low tree-depth coloring and $p$-centered colorings 26

4.6. Algorithmic Considerations 28

Supported by grant 1M0021620808 of the Czech Ministry of Education and AEOLUS. 
5. Algorithmic Applications 31

5.1. Subgraph Isomorphism Problem 31

5.2. Small Distance Checking 32

5.3. Existential First-order Properties 32

5.4. Dominating Sets 33

5.5. Induced Matchings 35

5.6. Vertex Separators 36

6. Homomorphisms and Logic 37

6.1. Restricted Dualities 38

6.2. Homomorphism Preservation 41

6.3. Richness of First Order 45

7. Summary (Characterization Theorems) 46

7.1. Polynomial Dependence 46

7.2. Characterizations 46

References 48

\section{Introduction}

In this chapter we briefly outline the main motivation our work and we relate it to other research. We do not include any definitions here.

\subsection{Dense Graphs}

Dense graphs have been extensively studied in the context of Extremal Graph Theory. The outstanding Szemerédi Regularity Lemma [111] states that any dense network has properties which are close to the ones of a random graph. In particular, a large dense network cannot be too irregular. This structural result is one of the cornerstones of contemporary combinatorics (and one would like to say mathematics in general). It also let to manyfold applications and generalizations, see e.g. [66, 65, 72, 113, 41]. The closest to our topic covered in this paper is the recent development which is based on the study of homomorphisms of graphs (and structures). (It is perhaps of interest note in how many different areas and a variety contexts the notion of a homomorphism recently appeared, see [60]). Regularity is viewed here as a sructural approximation in a proper metrics and also as 
a convergence. For a survey of this development see [15]. The main idea here is to study the local structure of a large graph $G$ by counting the homomorphisms from various small graphs $F$ into $G$ (this relates to the area called property testing), and to study the global structure of $G$ by counting its homomorphisms into various small graphs $H$ (sometimes interpreted as templates). Very schematically this may be outlined by the schema:

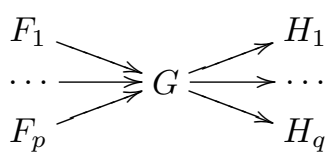

This approach proved to be very fruitful and relates (among others) to the notion of quasi-random graph, see e.g. [23], and to the full characterizations of testable graph properties, see e.g. [5, 15]. Nevertheless, such an approach fails when the considered structures become too sparse. In particular, Szemerédi's regularity lemma concerns graphs which have (at least locally) a number $m$ of edges which is quadratic with respect to the number $n$ of vertices, or at least as large as $n^{1+\epsilon}$ if one consider extensions and generalizations of this lemma to the sparse context, see e.g. [65]. It is our ambition to deal exactly with sparse graphs which are not covered by this spectrum of results. Yet our goals are similar: we are aiming for regular and highly regular partitions.

\subsection{Sparse Graphs}

We aim (as in the Szemerédi regularity lemma) for structural theorems for all graphs. The dense graphs display a remarkable stability (and many of their properties do not change by deletions and additions of a small proportion of all edges, see e.g. [117]) and, as has been discovered recently, they may be studied by number of homomorphisms and by limit objects of geometrical nature, $[15,41]$. But our graphs have typically linearly many edges, large independent sets and exponentially many endomorphism. As a consequence we do not consider statistical properties but rather existential properties, i.e. properties defined by the existence (and non-existence) of mappings. In the other words we deal with the simplification of category of graphs (or the homomorphism order), see e.g. [60]. But the first difficulty we shall meet is the definition of what a "sparse graph" is. Let us consider various approaches to this problem.

Of course, if we consider any dense graph and break every link by inserting a new vertex, the obtained graph has a number of links less than 
twice its number of vertices and nevertheless inherit most of the structure of the original graph. So the degeneracy (or maximal average degree of a subgraph) of our graphs is not sufficient. This also indicates that tests which contain a bounded number vertices are not sufficient to our purposes.

Another possible (and finer) restriction is to consider graphs with no minor belonging to some fixed family. In this way we get for example the class of all planar graphs. The interest of such a restriction is twofold: first it ensures a number of efficient algorithms, and also a large scientific literature. One way of describing such a family is the following: if you consider disjoint connected parts, you will never be able to find more than (fixed) $p$ parts which are pairwise adjacent. In the other words the complete graph $K_{p+1}$ is a forbidden minor. Classes like that are called proper minor closed. Such restrictions are natural for geometric networks, but for our purposes do not seem to be general enough. For instance, a very simple operation which is to clone every node (with its links) does not preserve such properties. Another feature is the lack of parametrization: one graph is "forbidden" at all levels.

Another interesting restriction is to consider bounded degree graphs. Such graphs almost surely have nice properties when large (they are almost surely expanders). Nevertheless, important real networks like the WEB surely does not fit this restriction. And this class does not include even the class of all trees (which should be considered as sparse graphs).

A more general framework (a framework which include the above examples) concerns proper topologically closed classes of graphs. These classes are characterized as follows: whenever a subdivision of a graph $G$ belongs to the class then $G$ belongs to the class; moreover, not every graph belong to the class. Such classes are obviously defined by a (maybe infinite) set of forbidden configurations. These classes naturally catch the classes from geometrical origin, and also appear as a good approximation base for real-world networks. Notice that such graphs still have a number of edges which is bounded by a linear function of their orders. But still this lacks a parametrization and our classes will strictly include these classes.

Our principal notion for sparse graphs is the notion of bounded expansion class of graphs. These classes are characterized by the fact that the average degree of minors obtained by contracting disjoint subgraphs each of radius at most $r$ is bounded by a function of $r$ only. This means that local contractions cannot make the graphs too dense. These classes will be introduced in detail in the next chapter and we shall also indicate the various equivalent definition and regularity properties of graphs belonging to these classes. 
The charecterization theorems are then summarized in the last chapter of this article.

\subsection{Nowhere Dense Graphs}

For any class with bounded expansion all graphs in the class have linear number of edges. There is numerous evidence that graphs with $n^{1+\epsilon}$ edges share many properties of random graphs (for example such graphs include graphs with large girth and high chromatic number, a seminal result of Erdős). Thus $n^{1+\epsilon}$ edges of a graph with $n$ vertices seems to be a natural bound for our investigations of sparse graphs. This bound is natural. As we will show (and motivated by problems from model theory) a new type of graph classes arises here: classes of nowhere dense graphs. These classes are characterized by the fact that the number $m$ of edges of a graph in the class is bounded by $n^{1+o(1)}$, where $n$ is the order of the graph, and that such a statement holds for the class of the minors obtained by contracting disjoint balls of radius at most $r$ for each fixed $r$. Again, this definition should be compared with the fact that every sufficiently big graph $G$ having at least $n^{1+\epsilon}$ edges has a big dense minor obtained by contracting balls of radius at most $r(\epsilon)$ (by dense we mean: having a quadratic number of edges). But not only that; the classes of nowhere dense graphs have a characterization which combines virtually all concepts which were developed for the study of bounded expansion classes and expose them in the new light. To demonstrate this explicitely we included all characterization theorem in the final section of this article.

\section{Measuring sparsity}

The distance in a graph $G$ between two vertices $x$ and $y$ is the minimum length of a path linking $x$ and $y$ (or $\infty$ if $x$ and $y$ do not belong to the same connected component of $G$ ) and is denoted by $\operatorname{dist}_{G}(x, y)$. Let $G=(V, E)$

be a graph and let $d$ be an integer. The $d$-neighborhood $N_{d}^{G}(u)$ of a vertex $u \in V$ is the subset of vertices of $G$ at distance at most $d$ from $u$ in $G$ : $N_{d}^{G}(u)=\left\{v \in V: \operatorname{dist}_{G}(u, v) \leq d\right\}$.

We use standard graph theory terminology however we find it useful to introduce the following: for a graph $G=(V, E)$, we denote by $|G|$ the order of $G$ (that is: $|V|$ ) and by $\|G\|$ the size of $G$ (that is: $|E|$ ). 


\subsection{Shallow MiNORS AND Grads}

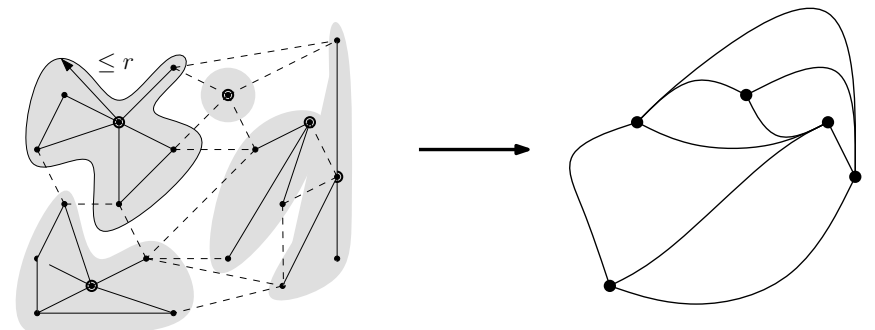

Figure 2.1. A shallow minor of depth $r$ of a graph $G$ is a simple subgraph of a minor of $G$ obtained by contracting vertex disjoints subgraphs with radius at most $r$

For any graphs $H$ and $G$ and any integer $d$, the graph $H$ is said to be a shallow minor of $G$ at depth $d$ ([100] attributes this notion, then called low depth minor, to Ch. Leiserson and S. Toledo) if there exists a subset $\left\{x_{1}, \ldots, x_{p}\right\}$ of $G$ and a collection of disjoint subsets $V_{1} \subseteq N_{d}^{G}\left(x_{1}\right), \ldots, V_{p} \subseteq$ $N_{d}^{G}\left(x_{p}\right)$ such that $H$ is a subgraph of the graph obtained from $G$ by contracting each $V_{i}$ into $x_{i}$ and removing loops and multiple edges (see Fig. 2.1). The set of all shallow minors of $G$ at depth $d$ is denoted by $G \nabla i$. In particular, $G \nabla 0$ is the set of all subgraphs of $G$.

The greatest reduced average density (shortly grad) with rank $r$ of a graph $G[88]$ is defined by formula

$$
\nabla_{r}(G)=\max \left\{\frac{\|H\|}{|H|}: H \in G \nabla r\right\}
$$

Also we denote by $\nabla(G)=\nabla_{\infty}(G)$ the maximum edge-density of a minor of $G$. Notice that this last invariant is related to the order of the largest complete graph which is a minor of $G$, that is: the so-called Hadwiger number $\eta(G)$ of $G$. It follows from the definition that

$$
\eta(G) \leq 2 \nabla(G)+1 .
$$

By extension, for a class of graphs $\mathcal{C}$, we denote by $\mathcal{C} \nabla i$ the set of all shallow minors at depth $i$ of graphs of $\mathcal{C}$, that is:

$$
\mathcal{C} \nabla i=\bigcup_{G \in \mathcal{C}}(G \nabla i)
$$


Hence we have

$$
\mathcal{C} \subseteq \mathcal{C} \nabla 0 \subseteq \mathcal{C} \nabla 1 \subseteq \cdots \subseteq \mathcal{C} \nabla i \subseteq \cdots \subseteq \mathcal{C} \nabla \infty
$$

Here we denoted by $C \nabla \infty$ the class of all minors of graphs from $\mathcal{C}$. This is of course a minor closed class of graphs (which may coincide with the class of all finite simple graphs; think e.g. of the class of all cubic graphs).

Also, for a class $\mathcal{C}$ of graphs we define the expansion of the class $\mathcal{C}$ as:

$$
\begin{aligned}
\nabla_{i}(\mathcal{C}) & =\sup _{G \in \mathcal{C}} \nabla_{i}(G) \\
\nabla(\mathcal{C}) & =\sup _{G \in \mathcal{C}} \nabla(G)
\end{aligned}
$$

Notice that $\nabla_{r}(G)=\nabla_{0}(G \nabla r)$.

A proper minor closed class of graphs $\mathcal{C}$ is a minor closed class of graphs excluding at least one minor, i.e. such that $\mathcal{C}$ is not the class of all finite simple graphs. Every proper minor closed class of graphs $\mathcal{C}$ is such that $\nabla(\mathcal{C})<\infty$. Conversely, if $\mathcal{C}$ is a class of graphs such that $\nabla(\mathcal{C})<\infty$ then $\mathcal{C}$ is a subclass of a proper minor closed class of graphs (the smallest being $\mathcal{C} \nabla \infty)$.

Also, a grad of particular importance is $\nabla_{0}$. It is related to the maximum average degree (mad) of a graph by $\operatorname{mad}(G)=2 \nabla_{0}(G)$. A class $\mathcal{C}$ of graphs such that $\nabla_{0}(G)<(k+1) / 2$ (where $k$ is an integer) is called $k$-degenerate. The equivalent defining property of a $k$-degenerate class of graphs is that every non-empty subgraph contains at least a vertex of degree at most $k$. Thus there is also an easy (greedy) algorithm to determine $\nabla_{0}(G)$.

It has to be noticed [38] that the determination of $\nabla_{r}(G)$ is a difficult problem whenever $r \geq 1$.

\subsection{Shallow Topological Minors and Top-grads}

Our approach makes it possible to treat minors and topological subgraphs similarly. For any (simple) graphs $H$ and $G$ and any integer $d$, the graph $H$ is said to be a shallow topological minor of $G$ at depth $d$ if there exists a subset $\left\{x_{1}, \ldots, x_{p}\right\}$ of $G$ and a collection of internally vertex disjoint paths $P_{1} \ldots P_{q}$ each of length at most $d+1$ of $G$ with endpoints in $\left\{x_{1}, \ldots, x_{p}\right\}$ whose contraction into single edges define on $\left\{x_{1}, \ldots, x_{p}\right\}$ a graph isomorphic to $H$ (see Fig. 2.2).

The set of all the shallow topological minors of $G$ at depth $d$ is denoted by $G \widetilde{\nabla} i$. In particular, $G \widetilde{\nabla} 0$ is the set of all the subgraphs of $G$. Notice that for every graph $G$ and every integer $i$ we clearly have $(G \widetilde{\nabla} i) \subseteq(G \nabla i)$. 


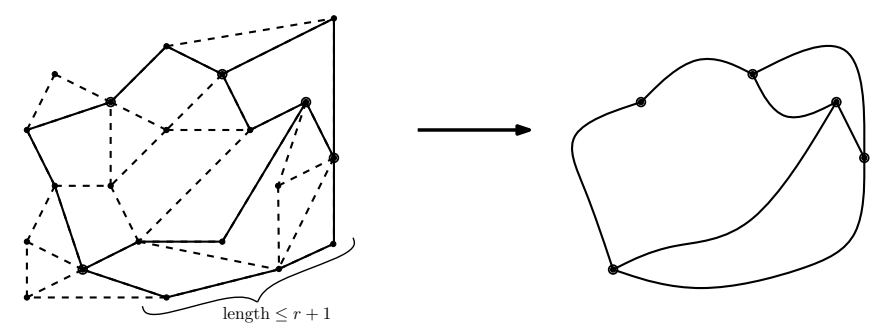

Figure 2.2. A shallow topological minor of depth $r$ of a graph $G$ is a simple subgraph of a minor of $G$ obtained by contracting internally vertex disjoint paths of length at most $r+1$

The topological greatest reduced average density (top-grad) with rank $r$ of a graph $G$ is:

$$
\widetilde{\nabla}_{r}(G)=\max \left\{\frac{\|H\|}{|H|}: H \in G \widetilde{\nabla} r\right\}
$$

Also, we denote by $\widetilde{\nabla}(G)$ the limit value $\widetilde{\nabla}_{\infty}(G)$.

By extension, for a class of graphs $\mathcal{C}$, we denote by $\mathcal{C} \widetilde{\nabla} i$ the set of all shallow topological minors at depth $i$ of graphs of $\mathcal{C}$, that is:

$$
\mathcal{C} \widetilde{\nabla} i=\bigcup_{G \in \mathcal{C}}(G \widetilde{\nabla} i)
$$

Hence we have

$$
\mathcal{C} \subseteq \mathcal{C} \widetilde{\nabla} 0 \subseteq \mathcal{C} \widetilde{\nabla} 1 \subseteq \cdots \subseteq \mathcal{C} \widetilde{\nabla} i \subseteq \cdots \subseteq C \widetilde{\nabla} \infty
$$

For a class $\mathcal{C}$ of graphs we define the topological expansion of $\mathcal{C}$ as:

$$
\begin{aligned}
\widetilde{\nabla}_{i}(\mathcal{C}) & =\sup _{G \in \mathcal{C}} \widetilde{\nabla}_{i}(G) \\
\widetilde{\nabla}(\mathcal{C}) & =\sup _{G \in \mathcal{C}} \widetilde{\nabla}(G)
\end{aligned}
$$

Notice that $\widetilde{\nabla}_{i}(\mathcal{C})=\widetilde{\nabla}_{0}(\mathcal{C} \widetilde{\nabla} i)$.

Also, a class $\mathcal{C}$ is topologically closed if $\mathcal{C}=\mathcal{C} \widetilde{\nabla} \infty$. A topologically closed class $\mathcal{C}$ is proper if it is different from the class of all simple finite graphs. Notice that a class $\mathcal{C}$ is a subclass of a proper topologically closed class of graphs if and only if $\widetilde{\nabla}(\mathcal{C})<\infty$. 


\subsection{HAJÓS OR HADWIGER?}

Although any proper minor closed class of graphs is also a proper topologically closed class, the converse is not true. Also, some important properties which holds from the former do not hold for the latter. A striking example stands in the fundamental difference Hadwiger conjecture (which is at least satisfied by almost every graphs) and Hajós conjecture (which is satisfied by almost no graphs).

Hence it seems to be of great importance to decide whether we will choose to define the sparsity of a class of graphs using the grad or the top-grad. However, a bit surprisingly, this does not make a difference at all. This is expressed by the following result of Zdeněk Dvořák, [38]:

Theorem 2.1. For every integer $r$, the invariants $\nabla_{r}$ and $\widetilde{\nabla}_{r}$ are polynomially equivalent. Precisely, for every graph $G$ :

$$
\frac{1}{4}\left(\frac{\nabla_{r}(G)}{4}\right)^{\left.\frac{1}{(r+1)}\right)^{2}} \leq \widetilde{\nabla}_{r}(G) \leq \nabla_{r}(G)
$$

Similar correspondence as for edge density (expressed in terms of grads and top-grads) but also for clique number $\omega(G)$ :

Lemma $2.2([91])$. Let $r \in \mathbb{N}$. For any graph $G$ :

$$
\omega(G \widetilde{\nabla} r) \leq \omega(G \nabla r) \leq 2^{2^{r}-1}\left(\omega\left(G \widetilde{\nabla} \frac{9^{r+1}-5}{2}\right)\right)^{2^{r+1}}
$$

These two results are related by the following theorem, which has been proved by Z. Dvořák in his thesis [38]:

Theorem 2.3. For each $\epsilon(0<\epsilon \leq 1)$ there exist integers $n_{0}$ and $c_{0}$ and a real number $\mu>0$ such that every graph $G$ with $n \geq n_{0}$ vertices and minimum degree at least $n^{\epsilon}$ contains the c-subdivision of $K_{n^{\mu}}$ as a subgraph, for some $c \leq c_{0}$.

\subsection{Stability with Respect to Lexicographic Product}

Let $G, H$ be graphs. The lexicographic product $G \bullet H$ is defined by

$V(G \bullet H)=V(G) \times V(H)$

$E(G \bullet H)=\left\{\left\{(x, y),\left(x^{\prime}, y^{\prime}\right):\left\{x, x^{\prime}\right\} \in E(G)\right.\right.$ or $x=x^{\prime}$ and $\left.\left\{y, y^{\prime}\right\} \in E(H)\right\}$.

Note that the lexicographic product (or blowing up of vertices) is incompatible with minors, since it is easily seen that every graph is a minor of 


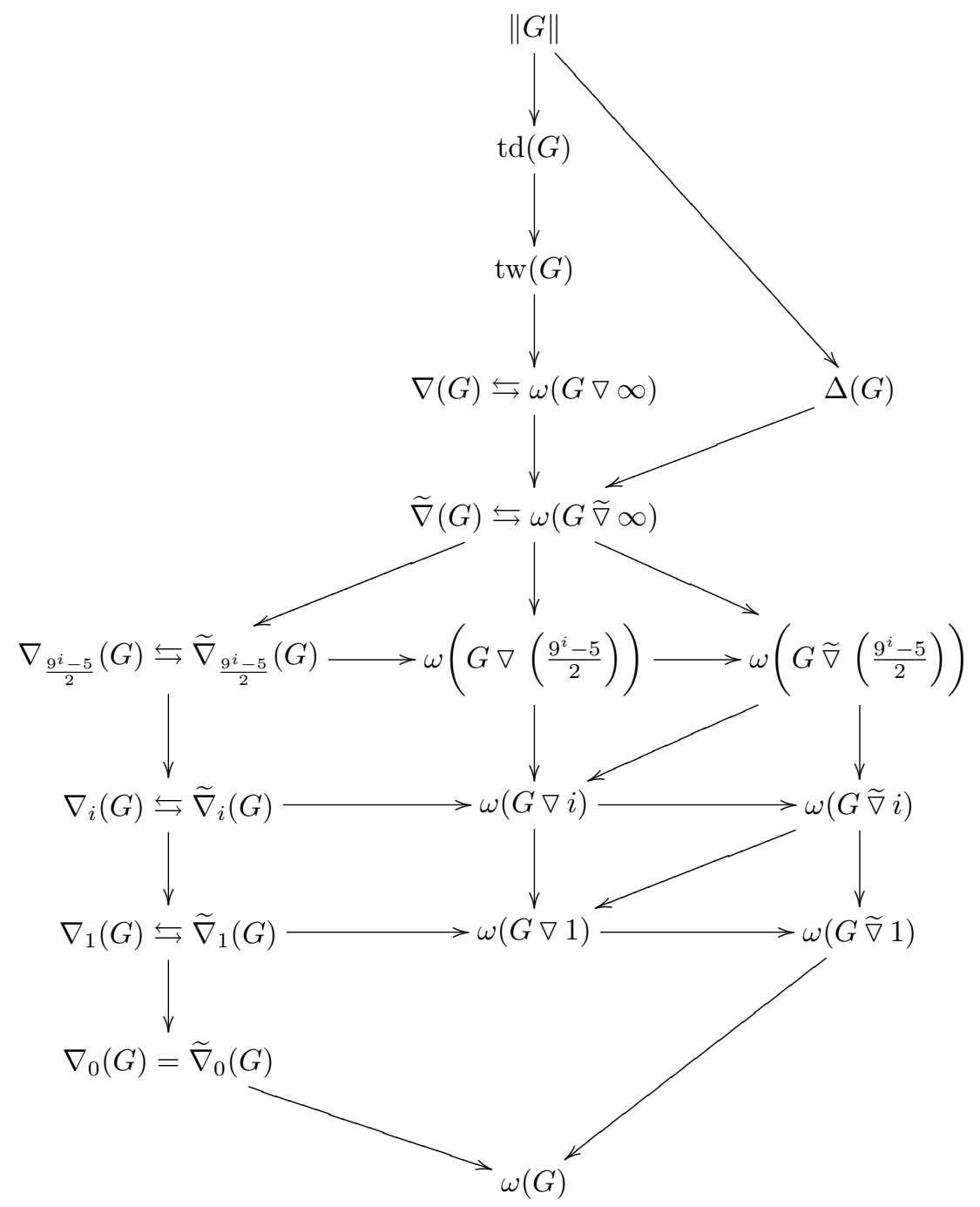

FiguRE 2.3. Dominance of Invariants (up to polynomial transformation) 
$G \bullet K_{2}$ for some planar graph $G$. However the lexicographic product and blow-up are natural constructions inthe context of homomorphisms and quasi-randomness.

The long and difficult proof of the following Lemma is omitted here.

Lemma 2.4 ([88]). For every integer $r$ there exists a polynomial $P_{r}$ of degree $O\left(\frac{(2 r+1) !}{2^{r} r !}\right)$ such that for every graphs $G$ and $H$ :

$$
\nabla_{r}(G \bullet H) \leq P_{r}\left(|H|, \nabla_{r}(G)\right) .
$$

We may notice a slight difference between the treatment of dense and sparse graphs: In the case of dense graphs, it is usual to consider that any blow-up of the vertices of a graph $G$ produce a graph which is intrinsically equivalent to $G$ (hence the definition of the distance in [15]). However, in the sparse case, we only allow to blow the vertices of the graphs a bounded number of times, and the obtained graphs although not "equivalent" have characteristic which are polynomially equivalent to the ones of the original graph.

Also, Lemma 2.4 is the core of the proof of the existence of bounded transitive fraternal augmentations for graphs with bounded grads, the heart of our decomposition result for sparse graphs (see Section 3.6).

Notice also that we have an easy inequality the other way:

Lemma 2.5. For every integer $r$ and for every graphs $G$ and $H$ :

$$
\nabla_{r}(G \bullet H) \geq \nabla_{r}(G)|H|
$$

Hence, for fixed $r, \nabla_{r}(G)|H|$ and $\nabla_{r}(G \bullet H)$ are polynomially equivalent.

Proof. Consider a shallow minor $G^{\prime}$ of $G$ of depth $r$ such that $\nabla_{r}(G)=\frac{\left\|G^{\prime}\right\|}{\left|G^{\prime}\right|}$. Then $G^{\prime} \bullet H$ is obviously a minor of $G \bullet H$ and $\frac{\left\|G^{\prime} \bullet H\right\|}{\left|G^{\prime} \bullet H\right|} \geq \frac{|H|^{2}\left\|G^{\prime}\right\|}{|H|\left|G^{\prime}\right|}=$ $|H| \frac{\left\|G^{\prime}\right\|}{|G|}$.

\section{Sparse Classes of Graphs}

\subsection{Basic Definitions}

A class $\mathcal{C}$ of graphs is hereditary if every induced subgraph of a graph in $\mathcal{C}$ to $\mathcal{C}$, and it is monotone of every subgraph of a graph in $\mathcal{C}$ belongs to $\mathcal{C}$. For a class of graphs $\mathcal{C}$, we denote by $H(\mathcal{C})$ the class containing all the 
induced subgraphs of graphs in $\mathcal{C}$, that is the inclusion-minimal hereditary class of graphs containing $\mathcal{C}$.

3.1.1. Limits. Let $\mathcal{C}$ be an infinite class of graphs and let $f: \mathcal{C} \rightarrow \mathbb{R}$ be a graph invariant. Let $\operatorname{Inj}(\mathbb{N}, \mathcal{C})$ be the set of all injective mappings from $\mathbb{N}$ to $\mathcal{C}$. Then we define:

$$
\limsup _{G \in \mathcal{C}} f(G)=\sup _{\phi \in \operatorname{Inj}(\mathbb{N}, \mathcal{C})} \limsup _{i \rightarrow \infty} f(\phi(i))
$$

Notice that $\lim \sup _{G \in \mathcal{C}} f(G)$ always exist and is either a real number or $\pm \infty$.

If $\lim \sup _{G \in \mathcal{C}} f(G)=\alpha \in \overline{\mathbb{R}}=\mathbb{R} \cup\{-\infty, \infty\}$ we have the following two properties:

- for every $\phi \in \operatorname{Inj}(\mathbb{N}, \mathcal{C}), \lim \sup _{i \rightarrow \infty} f(\phi(i)) \leq \alpha$;

- there exists $\phi \in \operatorname{Inj}(\mathbb{N}, \mathcal{C}), \lim \sup _{i \rightarrow \infty} f(\phi(i))=\alpha$.

The second property is easy to prove: consider a sequence $\phi_{1}, \ldots, \phi_{i}, \ldots$ such that $\lim _{i \rightarrow \infty} \limsup \sup _{j \rightarrow \infty} f\left(\phi_{i}(j)\right)=\alpha$. For each $i$, let $s_{i}(1)<\cdots<$ $s_{i}(j)<\ldots$ be such that $\limsup _{j \rightarrow \infty} f\left(\phi_{i}(j)\right)=\lim _{j \rightarrow \infty} f\left(\phi_{i}\left(s_{i}(j)\right)\right)$. Then iteratively define $\phi \in \operatorname{Inj}$ by $\phi(1)=\phi_{1}\left(s_{1}(1)\right)$ and $\phi(i)=\phi_{i}\left(s_{i}(j)\right)$, where $j$ is the minimal integer greater or equal to $i$ such that $\phi_{i}\left(s_{i}(j)\right)$ will be different from $\phi(1), \ldots, \phi(i-1)$. Then $\limsup _{j \rightarrow \infty} f(\phi(j))=\alpha$.

3.1.2. Derived classes. Graph operations naturally define operations on graph classes: for a class $\mathcal{C}$, an integer $r$ and a graph $H$, we define:

$$
\begin{aligned}
\mathcal{C} \nabla r & =\bigcup_{G \in \mathcal{C}} G \nabla r \\
\mathfrak{B}_{r}(\mathcal{C}) & =\{G \in \mathcal{C} \nabla 0: \rho(G) \leq r\} \\
\mathcal{C} \bullet H & =\{G \bullet H: G \in \mathcal{C}\} \\
\mathcal{C}+H & =\{G+H: G \in \mathcal{C}\}
\end{aligned}
$$

(Here $G+H$ ofcourse means the disjoint union of graphs $G$ and $H$.)

\subsection{When is a Class Sparse or Dense?}

Defining the boundary between sparse and dense classes is not an easy task. Several definitions have been given for "sparse graphs", which do not allow a dense/sparse dichotomy (for instance: a graph is sparse if it has a size which is linear with respect to its order, dense if it is quadratic). Instead 
of defining what is a "sparse graph" or a "dense graph", we define "sparse classes of graphs" and "dense classes of graphs" by the limit behaviour of the "biggest" graphs in the class when their order tends to infinity. Moreover, we will demand that our definition stays invariant in the context of derived classes, i.e. when we perform lexicographic products with small graphs, contractions of small balls, etc. It appears that the right measure of the growth of edge densities is the fraction of logarithms. This leads to the following trichotomy which is the starting point of our classification:

Lemma 3.1 ([91]). Let $\mathcal{C}$ be an infinite class of graphs. Then

$$
\lim _{r \rightarrow \infty} \limsup _{G \in \mathcal{C} \nabla r} \frac{\log \|G\|}{\log |G|} \in\{0,1,2\}
$$

The first case of Lemma 3.1, that is: $\lim _{r \rightarrow \infty} \lim \sup _{G \in \mathcal{C} \nabla r} \frac{\log \|G\|}{\log |G|}=0$ corresponds to a class of graphs $\mathcal{C}$ such that the number of edges of the graphs in $\mathcal{C}$ is bounded (for otherwise $\lim \sup _{G \in \mathcal{C} \nabla 0} \frac{\log \|G\|}{\log |G|}>0$ ). Then the graphs in $\mathcal{C}$ only contain isolated vertices with the exception of a bounded number of vertices. We say that such a class is a class of bounded size graphs.

The third case of Lemma 3.1, that is: $\lim _{r \rightarrow \infty} \lim \sup _{G \in \mathcal{C} \nabla r} \frac{\log \|G\|}{\log |G|}=2$ corresponds to a class of graphs $\mathcal{C}$ such that by considering shallow minors at some "reasonable" depth, one will find infinitely many dense graphs. Actually (as shown in [91]) the property of such classes is even stronger: there exists some threshold integer $r_{\mathcal{C}}$ such that $\mathcal{C} \nabla r_{\mathcal{C}}$ contains all finite graphs! Such classes we call classes of somewhere dense graphs.

Between these two extreme cases which seem to be well characterized lie the classes $\mathcal{C}$ such that:

$$
\lim _{r \rightarrow \infty} \limsup _{G \in \mathcal{C} \nabla r} \frac{\log \|G\|}{\log |G|}=1
$$

Such classes we call classes of nowhere dense graphs. They are alternatively defined by the fact that there exists no integer $r$ such that $\mathcal{C} \nabla r$ contains all finite graphs (i.e. such that $\omega(\mathcal{C} \nabla r)=\infty$ ). The intristic structure of this class and of its subclasses is the main subjet of this paper. The situation is summarized in the following diagram: 


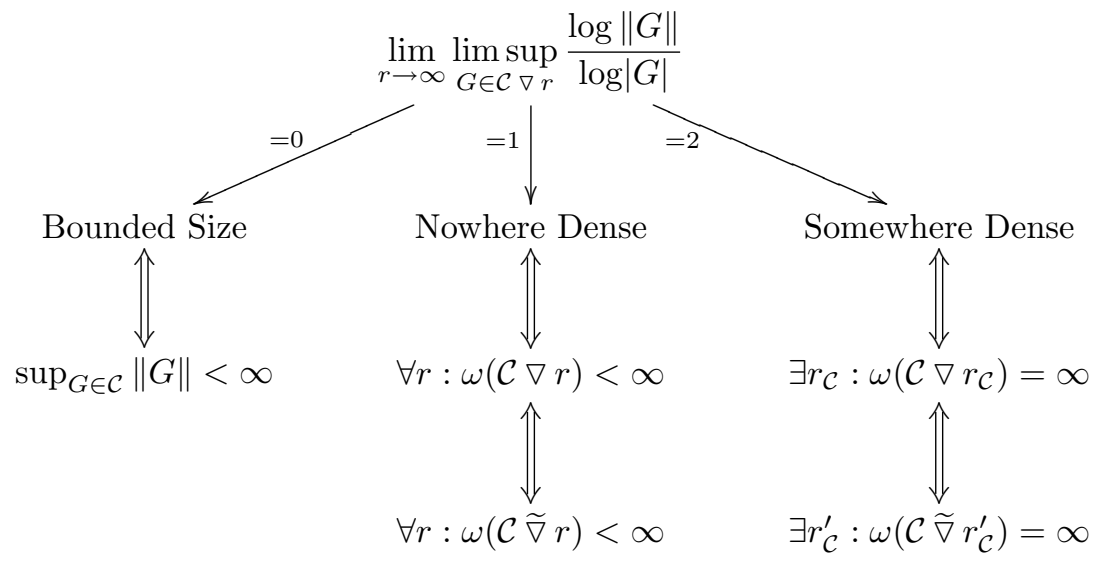

\subsection{Within the Nowhere Dense World}

Why do we have to consider shallow minors (i.e. classes $\mathcal{C} \nabla r$ )? Couldn't be a possible way to classify classes of nowhere dense graphs to look precisely at the behaviour of $\frac{\log \|G\|}{\log |G|}-1$ for $G \in \mathcal{C}$ and $|G| \rightarrow \infty$ ? Alas, it happens that this value can be equivalent to any function of $|G|$ which tends to zero:

Lemma 3.2. Let $\epsilon: \mathbb{N} \rightarrow \mathbb{R}$ be a function such that $\epsilon(n)>0$ and $\lim _{n \rightarrow \infty} \epsilon(n)=$ 0 . Then there exists an infinite hereditary class of nowhere dense graphs $\mathcal{C}_{\epsilon}$ such that

$$
\limsup _{G \in \mathcal{C}}\left(\frac{\log \|G\|}{\log |G|}-1\right) \sim \epsilon(|G|)
$$

Proof. We can use well known constructions of expanders and even weaker construction of [110], where a deterministic algorithm is given that constructs a graph of girth $\log _{k}(n)+O(1)$ and minimum degree $k-1, n$ is the number of vertices and number of edges is $e=\lfloor n k / 2\rfloor$ (where $k<\frac{n}{3}$ ). The degree of each node is guaranteed to be $k-1, k$, or $k+1$, where $k$ is the average degree.

As $\lim _{n \rightarrow \infty} \epsilon(n)=0$, there exists $N \in \mathbb{N}$ such that $\epsilon(N)<1$ and $N^{\epsilon(N)}<$ $N / 3$. For $n \geq N$, let $G_{n}$ be a graph of order $n$, average degree $n^{\epsilon(n)}$ and girth $g_{n}=\frac{1}{\epsilon(n)}+O(1)$. Let $\mathcal{C}=\left\{G_{n}\right\}_{n \in \mathbb{N}} \nabla 0$.

For $n, p, r \in \mathbb{N}$, assume $K_{p} \in G_{n} \widetilde{\nabla} r$. Then the girth of $G_{n}$ is at most $3(2 r+1)$ hence $\frac{1}{\epsilon(n)}+O(1) \geq 6 r$ thus $n \leq h(r)$ for some function $h: \mathbb{N} \rightarrow \mathbb{N}$. 
As obviously $p \leq n$ we deduce $p \leq h(r)$. It follows that $\omega(\mathcal{C} \widetilde{\nabla} r) \leq h(r)$ hence $\mathcal{C}$ is a class of nowhere dense graphs.

Hence we will be more modest in our tentative classification: we will base the classification on the rough behaviour of $\mathcal{C}$ with respect to bounded depth contractions. From the fact that $\lim _{r \rightarrow \infty} \limsup _{G \in \mathcal{C} \nabla r} \frac{\log \|G\|}{\log |G|}=1$, we can prove that the grads are "almost bounded" in the sense that $\nabla_{r}(G)=|G|^{o(1)}$ for $G \in \mathcal{C}$ and $|G| \rightarrow \infty$. This property suggest to consider the particular case where the function $\nabla_{r}(G)$ is actually bounded for every integer $r$. The classes for which $\nabla_{r}(G)$ is bounded by some value $f(r)$ independent of $G$ are called classes with bounded expansion. That is:

$$
\mathcal{C} \text { has bounded expansion } \Longleftrightarrow \forall i \geq 0: \sup _{G \in \mathcal{C}} \nabla_{i}(G)<\infty .
$$

The expansion of a class $\mathcal{C}$ with bounded expansion is the function $f$ defined by:

$$
f(r)=\nabla_{r}(\mathcal{C})=\sup _{G \in \mathcal{C}} \nabla_{r}(G)
$$

Let us remark here that we are explaining our definitions in the reverse chronologival order. Classes with bounded expansions were defined in 2005 (see e.g. [83, 84] while the importance of nowhere dense classes was realized recently (see e.g. [87]. An intermediate level between classes with bounded expansion and general classes of nowhere dense graphs are classes with bounded local expansion, defined by the fact that for every $\rho$, the class $\mathfrak{B}_{\rho}(\mathcal{C})$ of all balls of radius $\rho$ in graphs inC has bounded expansion. Alternatively, this may be expressed as follows:

$\mathcal{C}$ has bounded local expansion $\Longleftrightarrow \forall \rho, i \geq 0: \sup _{v \in G \in \mathcal{C}} \nabla_{i}\left(G\left[N_{\rho}^{G}(v)\right]\right)<\infty$.

The interest in these classes is limited by the fact that adding an apex to the graphs in the class destroy the property of a class to have bounded local expansion if it does not actually have a bounded expansion. However classes with bounded local expansion strictly contain classes with locally forbidden minors and they in turn minor closed classes. They were studied extensively, see e.g. [29]. A "standard example" of a class with bounded local expansion is the class $\mathcal{G}$ of graphs $G$ such that $\operatorname{girth}(G) \geq \Delta(G)$ : consider any fixed integer $r$ and the subgraph $G_{v}$ of $G \in \mathcal{G}$ induced by the $r$-neighborhood of $v$. Either $\Delta(G)<2 r$ and thus $\left|G_{v}\right| \leq(2 r)^{r}$ or $\Delta(G)>2 r$ thus $\operatorname{girth}(G)>2 r$ hence $G_{v}$ is a tree. Thus, except for a bounded number of graphs, the class $\mathfrak{B}_{r}(\mathcal{G})$ only includes forests. 
Another approach to sparsity is to look for subsets of vertices which are far away from each other. Intuitively, for any integer $d$, if a graph is sparse and sufficiently large it will be sufficient to delete few vertices to find a big subset of vertices, any two of which are at distance at least $d$. Such a deletion is necessary (as we shall see) if we don't want to restrict "sparsity" to "bounded degree".

Let $r \geq 1$ be an integer. A subset $A$ of vertices of a graph $G$ is $r$ independent if the distance between any two distinct elements of $A$ is strictly greater than $r$. We denote by $\alpha_{r}(G)$ the maximum size of an $r$-independent set of $G$. Thus $\alpha_{1}(G)$ is the usual independence number $\alpha(G)$ of $G$. A subset $A$ of vertices of $G$ is $d$-scattered if $N_{d}^{G}(u) \cap N_{d}^{G}(v)=\emptyset$ for every two distinct vertices $u, v \in A$. Thus $A$ is $d$-scattered if and only if it is $2 r$-independent.

A class of graphs is wide if every sufficiently large graph in the class contains an arbitrarily big $d$-scattered set. Following Dawar [28], a class if almost wide if deleting at most some number of vertices (bounded independently to $d$ ) makes it possible to find an arbitrarily big $d$-scattered set in a sufficiently large graph in the class. The class is quasi-wide classes when the number of vertices to delete may depend on $d$. Precisely:

$$
\begin{array}{rlrl}
\mathcal{C} \text { is wide } & \Longleftrightarrow & & \forall d \in \mathbb{N}, \liminf _{G \in \mathcal{C}} \alpha_{d}(G)=\infty \\
\mathcal{C} \text { is almost wide } & \Longleftrightarrow \exists s \in \mathbb{N}, & & \forall d \in \mathbb{N}, \liminf _{G \in \mathcal{C}} \max _{|S| \leq s} \alpha_{d}(G-S)=\infty \\
\mathcal{C} \text { is quasi wide } & \Longleftrightarrow \exists s: \mathbb{N} \rightarrow \mathbb{N}, & \forall d \in \mathbb{N}, \liminf _{G \in \mathcal{C}} \max _{|S| \leq s(d)} \alpha_{d}(G-S)=\infty
\end{array}
$$

It has been proved in [9] that classes with bounded degree are wide, and in [27] that proper minor closed classes of graphs are almost wide. In [87] we characterized these classes and showed how they relate to the classes of nowhere dense graphs. In particular, we prove that a hereditary class of graph is quasi-wide if and only if it is a class of nowhere dense graphs, see Section 5.2.

\subsection{Classes with Bounded Expansion}

For an extensive study of bounded expansion classes we refer the reader to [88] [89] [90] [38] [39].

Let us list some examples of classes with bounded expansion. Some inclusions of these classes are schematically depicted on Fig 3.1. However, 

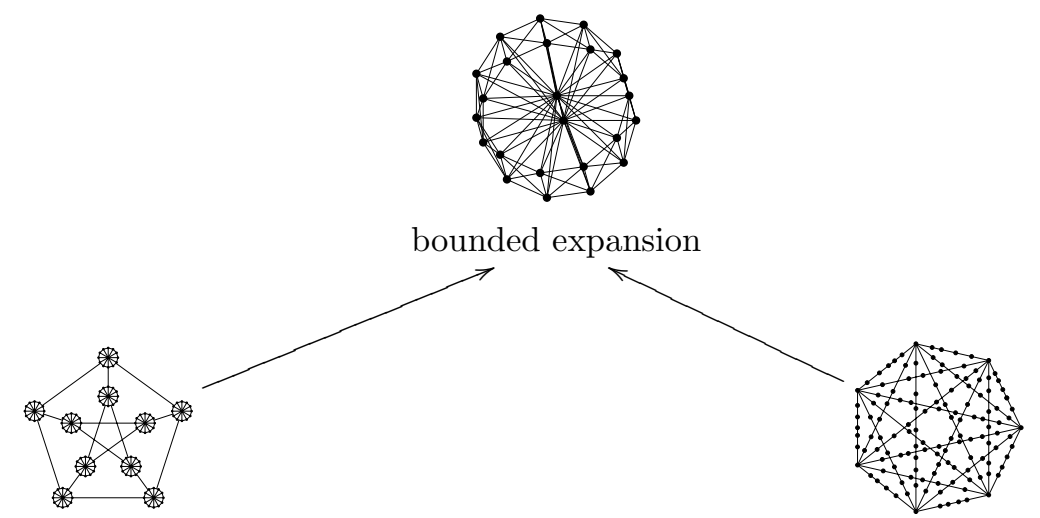

no $K_{p}$ subdivisions

highly subdivided

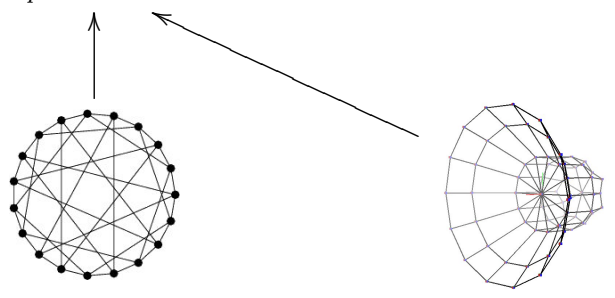

bounded degree

no $K_{p}$ minors

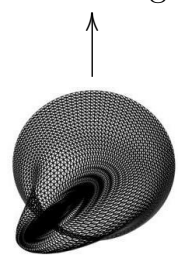

$d$-dimensional meshes

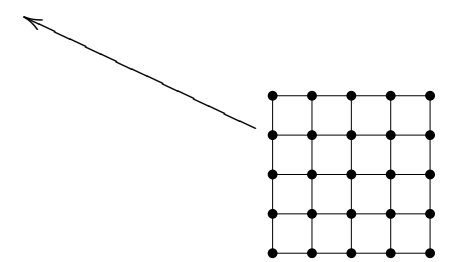

planar

with bounded aspect ratio

Figure 3.1. Classes with Bounded Expansion

we should remark that these classes may correspond to different expansion functions. 
- $d$-dimensional meshes with bounded aspect ratio. [75] introduces classes of graphs which occur naturally in finite-element and finite-difference problems. Theses classes, the classes of $d$ dimensional meshes with bounded aspect ratio, are formed by the interior skeletons of a family of $d$-dimensional simplicial complexes with bounded aspect ratio. As such graphs exclude $K_{h}$ as a depth $L$ minor if $h=\Omega\left(L^{d}\right)$ [114] they form (for each $d$ ) a class with polynomially bounded expansion. Our results (and particularly linear algorithm for low tree depth decompositions, see Sections 3.5 and 3.6) present a natural link of applicable results [75].

- bounded degree classes. Let $\Delta$ be an integer. Then the class of graphs with maximum degree at most $\Delta$ has expansion bounded by the exponential function $f(r)=\Delta^{r+1}$.

- planar graphs. Any planar graph graph $G$ of order $n$ has size at most $3 n-6$, hence $\nabla_{0}(G)<3$ for every planar graph. As any minor of a planar graph is also planar, $\nabla_{r}(G)<3$ for every integer $r \geq 0$ and any planar graph $G$. Hence the class of planar graphs has bounded expansion.

- proper minor closed classes. More generally, any proper minor closed class of graphs has expansion bounded by a constant function. Conversely, any class of graphs with expansion bounded by a constant is included in some proper minor closed class of graphs.

- proper topologically closed classes. These classes are defined by a (possibly infinite) set $\mathcal{S}$ of forbidden configurations, in the sense of Kuratowski's configurations: a graph $G$ belongs to the class if no subdivision of a graph in $\mathcal{S}$ is isomorphic to a subgraph of $G$. Such classes have expansion bounded by a double exponential function $f(r)=2^{r-1}\left(\min _{H \in \mathcal{S}}|V(H)|\right)^{2^{r+1}}$ (see [83]).

- highly subdivided cliques. For any non-decreasing function $f$ : $\mathbb{N} \rightarrow \mathbb{N} \backslash\{0,1,2\}$ we may construct a class $\mathcal{C}_{f}$ of graphs with expansion $f$ by including (for each integer $r$ ) the complete graph of $2 f(r)+1$ vertices whose edges are subdivided $3^{r}-1$ times.

- union of bounded expansion classes. Union of finitely many classes each with bounded expansion is itself a class with bounded expansion.

\subsection{Proper Minor Closed Classes}

Minor closed classes have been extensively studied by Robertson and Seymour (see [102] for instance). From our point of view, proper minor 
closed classes of graphs (that is: minor closed classes excluding at least one minor) form the very extreme case where the expansion of the class is uniformly bounded by a constant.

Important results have been obtained concerning proper minor closed graphs, such as the celebrated proof of Wagner's conjecture (the minor relation is a well quasi-order) and the Structure Theorem. This field is also strongly connected to the study of another fundamental conjecture, namely Hadwiger's conjecture.

In their study of classes of graphs excluding a minor, Robertson and Seymour have shown the particular importance of the tree-width $\operatorname{tw}(G)$ and of classes with bounded tree-width. Structural and algorithmic importance of tree-width [103] also appeared in the context of Monadic Second-order Logic (MSL) through the results of Courcelle [25] [26].

In [84], we introduced yet a more restrictive type of classes of graphs, related to a new invariant: the tree-depth $\operatorname{td}(G)$. Although a class of graphs has bounded tree-width if and only if it excludes some grid as a minor, it has bounded tree-depth if and only if it excludes some path as a minor. Classes with bounded tree-depth appear to behave like classes of "almost finite" graphs. For instance, only a bounded number of graphs with tree-depth at most fixed $k$ have no non-trivial involutive automorphism (see Section 3.2).

\subsection{The Full Picture}

The hierarchy of some important properties of hereditary sparse classes of graphs is depicted Fig. 3.2. It is interesting to note that all the properties shown in Fig. 3.2 are preserved when considering depth 1 shallow minors. This means that the considered properties are "weakly minor closed". For instance, $\mathcal{C}$ has bounded degree if and only if $\mathcal{C} \nabla 1$ has bounded degree. We give a short proof for the case of bounded local tree-width for completeness (a similar proof applies for locally excluded minors):

Lemma 3.3. Let $\mathcal{C}$ be a class of graphs. Then $\mathcal{C}$ has bounded local tree-width if and only if $\mathcal{C} \nabla 1$ has bounded local tree-width.

Proof. It is sufficient to prove that if $\mathcal{C}$ has bounded local tree-width, so has $\mathcal{C} \nabla 1$. Let $f: \mathbb{N} \rightarrow \mathbb{N}$ be such that for every connected $H \subseteq G \in \mathcal{C}$ and every $t \in \mathbb{N}$ we have $\rho(H) \leq t \Longrightarrow \operatorname{tw}(H) \leq f(t)$ (where $\rho(H)$ is the radius of $H$ ). Let $G \in \mathcal{C}$ and let $H \in G \nabla 1$. Then there is $G^{\prime} \subseteq G$ such that $H \in G^{\prime} \nabla 1$ and $\rho\left(G^{\prime}\right) \leq 3 \rho(H)$. As tw is minor-monotone, we deduce $\operatorname{tw}(H) \leq \operatorname{tw}\left(G^{\prime}\right) \leq f\left(\rho\left(G^{\prime}\right)\right) \leq f(3 \rho(H))$. It follows that $\mathcal{C} \nabla 1$ has bounded local tree-width. 


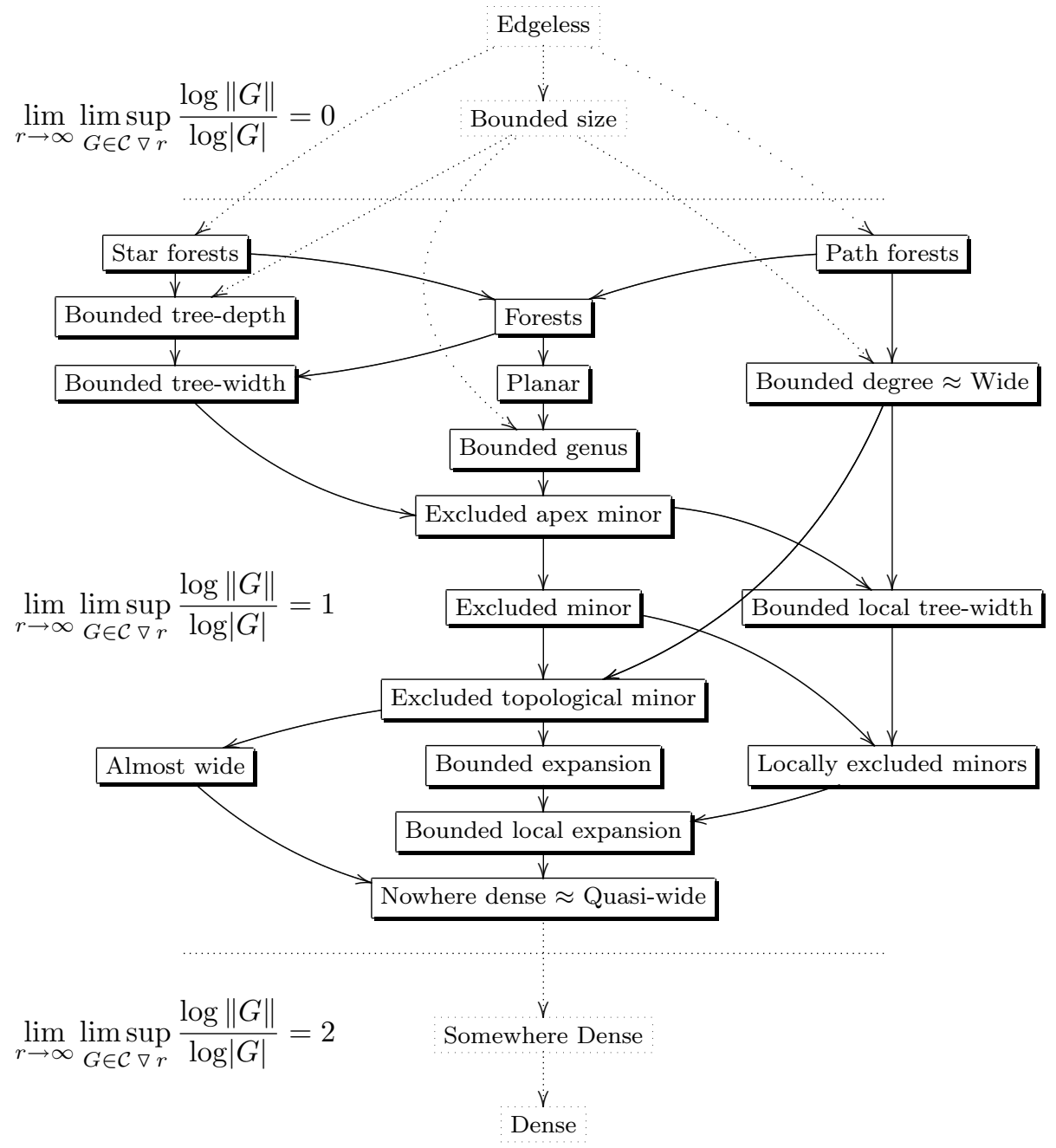

Figure 3.2. The Nowhere Dense World: inclusion map of some important properties of hereditary classes of graphs. 


\section{Regular Partitions of Sparse Graphs}

\subsection{TREe-WIDTH}

The concept of tree-width [59],[101],[116] is central to the analysis of graphs with forbidden minors of Robertson and Seymour. This concept gained much algorithmic attention thanks to the general complexity result of Courcelle about monadic second-order logic graph properties decidability for graphs with bounded tree-width [25],[26]. It appeared that many NPcomplete problems may be solved in polynomial time when restricted to a class with bounded tree-width. However, bounded tree-width is quite a strong restriction, as planar graphs for instance do not have bounded treewidth.

Let $k$ be an integer. A $k$-tree is a graph which is either a clique of size at most $k$ or a graph $G$ inductively constructed from a $k$-tree with order one less $G^{\prime}$ by adding a vertex adjacent to a clique of size at most $k$ of $G^{\prime}$. A partial $k$-tree is a subgraph of a $k$-tree. Although it is not the usual definition of tree-width (but is equivalent to it) we define the tree-width of a graph in terms of partial $k$-trees: The tree-width $\operatorname{td}(G)$ of a graph $G$ is the minimum $k$ such that $G$ is a partial $k$-tree. Notice that a graph $G$ with tree-width $k$ is $k$-degenerate is the sense that every non-empty subgraph of $G$ has at least one vertex of degree at most $k$ (this of course doesn'y hold conversely).

It is NP-complete to determine whether a given graph $G$ has tree-width at most a given variable $k$ [6]. However, when $k$ is any fixed constant, the graphs with tree-width $k$ can be recognized in linear time [13].

The notion of tree-width is closely related to the one of vertex-separator. An $\alpha$-vertex separator of a graph $G$ of order $n$ is a subset $S$ of vertices such that every connected component of $G-S$ contains at most $\alpha n$ vertices. It is proved in [103] that any graph of tree-width at most $k$ has a $\frac{1}{2}$-vertex separator of size at most $k+1$.

\subsection{TREE-DEPTH}

The concept of tree-depth has been introduced in [80] [84] to study generalized chromatic numbers of graphs (which will be introduced in Section $3.5)$.

A rooted forest is a disjoint union of rooted trees. The height of a vertex $x$ in a rooted forest $F$ is the number of vertices of a path from the root 
(of the tree to which $x$ belongs to) to $x$ and is noted height $(x, F)$. The height of $F$ is the maximum height of the vertices of $F$. Let $x, y$ be vertices of $F$. The vertex $x$ is an ancestor of $y$ in $F$ if $x$ belongs to the path linking $y$ and the root of the tree of $F$ to which $y$ belongs to. The closure $\operatorname{clos}(F)$ of a rooted forest $F$ is the graph with vertex set $V(F)$ and edge set $\{\{x, y\}: x$ is an ancestor of $y$ in $F, x \neq y\}$. A rooted forest $F$ defines a partial order on its set of vertices: $x \leq_{F} y$ if $x$ is an ancestor of $y$ in $F$. The comparability graph of this partial order is obviously $\operatorname{clos}(F)$.

The tree-depth $\operatorname{td}(G)$ of a graph $G$ is the minimum height of a rooted forest $F$ such that $G \subseteq \operatorname{clos}(F)$. This definition is analogous to the definition of rank function of a graph which has been used for analysis of countable graphs, see e.g. [95]. The concept also plays a key role in the recent beautifull proof of Rossmann [105].

The tree-depth of a graph may alternatively be defined inductively as follows: Let $G$ be a graph and let $G_{1}, \ldots, G_{p}$ be its connected components. Then

$$
\operatorname{td}(G)= \begin{cases}1, & \text { if }|V(G)|=1 ; \\ 1+\min _{v \in V(G)} \operatorname{td}(G-v), & \text { if } p=1 \text { and }|V(G)|>1 \\ \max _{i=1}^{p} \operatorname{td}\left(G_{i}\right), & \text { otherwise. }\end{cases}
$$

The tree-depth is minor monotone: if $H$ is a minor of $G$ then $\operatorname{td}(H) \leq$ $\operatorname{td}(G)$. The tree-depth $\operatorname{td}(G)$ of a graph $G$ is related to the order $l(G)$ of a longest path of $G$ by:

$$
l(G) \leq \operatorname{td}(G) \leq 2^{l(G)}
$$

and to its tree-width (see [84] [14]) by:

$$
\operatorname{tw}(G)+1 \leq \operatorname{td}(G) \leq(\operatorname{tw}(G)+1) \log _{2} n .
$$

The upper bound is, for instance, attained for paths (see Fig 4.1).

The tree-depth is also related to vertex-separators: for a graph $G$ of order $n$ and an integer $i \leq n$, let $s_{G}(i)$ be the maximum size of a $\frac{1}{2}$-vertex separator of a subgraph of $G$ of order at most $i$. Then:

$$
\operatorname{td}(G) \leq \sum_{i=1}^{\log _{2} n} s_{G}\left(\frac{n}{2^{i}}\right)
$$

what implies that every graph $G$ of order $n$ with no minor isomorphic to $K_{h}$ has tree-depth at most $(2+\sqrt{2}) \sqrt{h^{3} n}$ (as a graph of order $i$ with no $K_{h}$ minor has a $\frac{1}{2}$-vertex separator of size at most $\left.\sqrt{h^{3} i}[4]\right)$. 


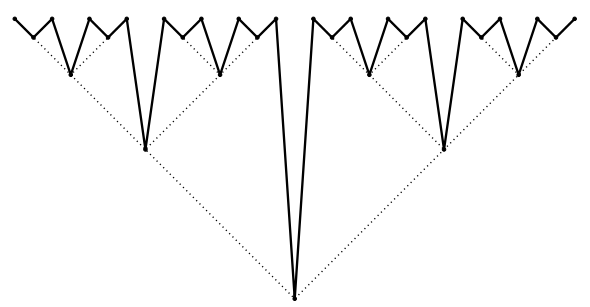

FiguRE 4.1. The tree-depth of a path is logarithmic in the order of the path

Although there is an (easy) polynomial algorithm to decide whether $t d(G) \leq k$ for any fixed $k$, if $\mathrm{P} \neq \mathrm{NP}$ then no polynomial time approximation algorithm for the tree-depth can guarantee an error bounded by $n^{\epsilon}$, where $\epsilon$ is a constant with $0<\epsilon<1$ and $n$ is the order of the graph [14].

One of the strongest properties of tree-depth is "finiteness" of graphs with bounded tree-depth. Precisely, there exists a function $\digamma: \mathbb{N} \times \mathbb{N} \rightarrow$ $\mathbb{N}$ with the following property: For any integer $N$, any graph $G$ of order $n>\digamma(N, \operatorname{td}(G))$ and any coloring $g: V(G) \rightarrow\{1, \ldots, N\}$, there exists a non trivial involuting $g$-preserving automorphism $\mu: G \rightarrow G$. As a consequence, any asymmetric (or rigid) graph of tree-depth $t$ has order at most $\digamma(1, t)$. Also, any graph $G$ is hom-equivalent to one of its induced subgraph of order at most $\digamma(1, \operatorname{td}(G))$. Hence the class $\mathcal{D}_{k}$ of all graphs $G$ with $\operatorname{td}(G) \leq k$ includes a finite subset $\hat{\mathcal{D}}_{k}$ such that, for every graph $G \in \mathcal{D}_{k}$, there exists $\hat{G} \in \hat{\mathcal{D}}_{k}$ which is hom-equivalent to $G$ and isomorphic to an induced subgraph of $G$.

The finitness is a deep property of finite structures which are "spanned" by a branching and it has many forms. For example we can consider the category of all pairs $(G, T)$ where $G$ is a graph (or a structure), $T$ a rooted tree (or branching) and $G \subseteq \operatorname{clos}(F)$. Such objects can be callet graphtr. The morphisms between graphtrs are mappings which preserve both edges of the graph and arcs of the branchings. The above results about involutory automorphisms and finitely many hom-equivalent objects hold also in this category. Many variations are possible, see also [105] where tree depth corresponds to the quantfier rank. At ts place we want to mention that the above function $\digamma$ grows very fast and it has Ackerman growth.

The tree-depth is intimately related to special types of colorings:

A centered coloring of a graph $G$ is a vertex coloring such that, for any (induced) connected subgraph $H$, some color $c(H)$ appears exactly once 
in $H$. Note that a centered coloring is necessarily proper. Actually, the minimum number of colors in a centered coloring of a graph $G$ is exactly $\operatorname{td}(G)[84]$.

We can also relate the minimum number of colors in a centered coloring to the notion of vertex ranking number which has been investigated in [30],[106]: The vertex ranking (or ordered coloring) of a graph is a vertex coloring by a linearly ordered set of colors such that for every path in the graph with end vertices of the same color there is a vertex on this path with a higher color. A vertex-coloring $c: V(G) \rightarrow\{1, \ldots, t\}$ with this property is a vertex t-ranking of $G$. The minimum $t$ such that $G$ has a vertex $t$-ranking is the vertex ranking number of $G$ (see [30],[106]). This parameter also equals $\operatorname{td}(G)[84]$.

\subsection{Generalized Coloring Numbers}

Consider the following ordering game played by Alice and Bob with Alice playing first. The players take turns choosing vertices from the set of unchosen vertices. This creates a linear order $L$ of the vertices of $G$ with $x<y$ if and only if $x$ is chosen before $y$. Given a linear order $L$ on $V$, the back degree of a vertex $x$ relative to $L$ is the number of neighbors of $x$ which precedes $x$ in $L$. The back degree of $L$ is the maximum back degree of a vertex relative to $L$. Alice's goal is to minimize the back degree of $L$, while Bob's goal is to maximize the back degree of $L$. This is a zero-sum two person game. Therefore each player has an optimal strategy. The game coloring number $\operatorname{col}_{g}(G)$ is the smallest (largest) integer $t$ for which Alice (Bob) has a strategy to ensure that the linear order produced by playing the game has back degree at most (at least) $t-1$.

For instance, the complete bipartite graph $K_{n, n}$ has game coloring number $n+1$. It was proved by Faigle et al. [47] that the game coloring number of a forest is at most 4, and that the game coloring number of an interval graph $G$ is at most $3 \omega-2$. It was proved by Zhu [118] that the game coloring number of the planar graphs is at most 19 and this bound has been further reduced by Kierstead to 18 [62] and by Zhu to 17 [121]. It has also been shown by Guan and Zhu [55] that the outerplanar graphs have game coloring number at most 7 . The game coloring number of graphs with bounded $\nabla_{1}$ is bounded (see [85], [121] p 3 and [38]).

As a generalization of both arrangeability and coloring number Kierstead and Yang introduced in [63] two new series of invariants $\mathrm{col}_{k}$ and $\mathrm{wcol}_{k}$, that is: the coloring number of rank $k$ and the weak coloring number of rank $k$. 
Let $L$ be a linear order on the vertex set of a graph $G$, and let $x, y$ be vertices of $G$. We say $y$ is weakly $k$-accessible from $x$ if $y<_{L} x$ and there exists an $x$-y-path $P$ of length at most $k$ (i.e. with at most $k$ edges) with minimum vertex $y$ with respect to $<_{L}$ (see Fig. 4.2). The vertex is $k$-accessible from $x$ if $y<_{L} x$ and there exists an $x$-y-path $P$ of length at most $k$ with minimum vertex $y$ and second minimum vertex $x$ with respect to $<_{L}$.

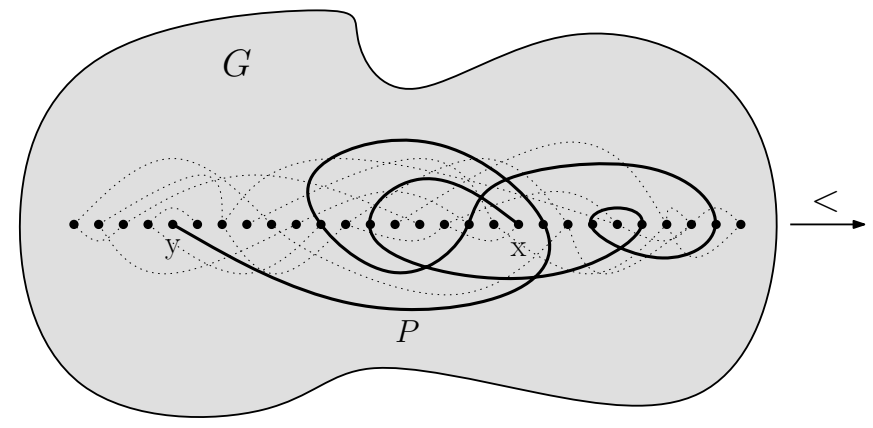

Figure 4.2. The vertex $y$ is weakly 8 -accessible from $x$

let $Q_{k}(G, L, x)$ and $R_{k}(G, L, x)$ be the sets of vertices that are respectively weakly $k$-accessible and $k$-accessible from $x$ :

$Q_{k}(G, L, x)=\{y: \exists x-y$ path $P$ such that $\min P=y\}$

$R_{k}(G, L, x)=\{y: \exists x-y$ path $P$ such that $\min P=y$ and $\min (P-y)=x\}$

The weak $k$-coloring number $\operatorname{wcol}_{k}(G)$ and the $k$-coloring number $\operatorname{col}_{k}(G)$ of $G$ are defined by:

$$
\begin{aligned}
\operatorname{wcol}_{k}(G) & =1+\min _{L} \max _{v \in V(G)}\left|Q_{k}(G, L, v)\right|, \\
\operatorname{col}_{k}(G) & =1+\min _{L} \max _{v \in V(G)}\left|R_{k}(G, L, v)\right| .
\end{aligned}
$$

These two graph invariants are polynomially dependent, as shown in [63]:

$$
\operatorname{col}_{k}(G) \leq \operatorname{wcol}_{k}(G) \leq\left(\operatorname{col}_{k}(G)\right)^{k}
$$

They form two non-decreasing sequences, the sequence of weak-coloring numbers having the tree-depth as its maximum:

$\operatorname{col}(G)=\operatorname{wcol}_{1}(G) \leq \operatorname{wcol}_{2}(G) \leq \cdots \leq \operatorname{wcol}_{k}(G) \leq \cdots \leq \operatorname{wcol}_{\infty}(G)=\operatorname{td}(G)$ 
Generalized coloring numbers are strongly related to grads: it has been proved by X. Zhu that there exists polynomials $F_{k}$ such that the following holds:

Theorem 4.1 ([120]). For every integer $k$ and every graph $G$ :

$$
\nabla_{\left\lfloor\frac{k-1}{4}\right\rfloor}(G) \leq \operatorname{wcol}_{k}(G) \leq F_{k}\left(\nabla_{\lfloor k / 2\rfloor}(G)\right)
$$

\subsection{LOW TREE-WIDTH COLORING}

A class $\mathcal{C}$ has a low tree-width coloring if, for any integer $p \geq 1$, there exists an integer $N(p)$ such that any graph $G \in \mathcal{C}$ may be vertex-colored using $N(p)$ colors so that each of the connected components of the subgraph induced by any $i \leq p$ parts has tree-width at most $(i-1)$. According to this definition, the result of DeVos et al. may be expressed as

Theorem 4.2 ([31]). Any minor closed class of graphs excluding at least one graph has a low tree-width coloring.

\subsection{LOW TREE-DEPTH COLORING AND $p$-CENTERED COLORINGS}

As we introduced low tree-width coloring, we say that a class $\mathcal{C}$ has a low tree-depth coloring if, for any integer $p \geq 1$, there exists an integer $N(p)$ such that any graph $G \in \mathcal{C}$ may be vertex-colored using $N(p)$ colors so that each of the connected components of the subgraph induced by any $i \leq p$ parts has tree-depth at most $i . \operatorname{As} \operatorname{td}(G) \geq \operatorname{tw}(G)-1$, a class having a low-tree depth coloring has a low tree-width coloring.

\begin{tabular}{|l|l|l|}
\hline \multicolumn{3}{|c|}{ Vertex Partitions } \\
\hline Parameter & \multicolumn{1}{|c|}{ Tree-Width } & \multicolumn{1}{c|}{ Tree-Depth } \\
\hline \hline 1 & \multicolumn{2}{|c|}{ proper coloring } \\
\hline 2 & $\begin{array}{l}\text { acyclic coloring [16] } \\
\text { low tree-width decom- } \\
\text { position [31] }\end{array}$ & $\begin{array}{l}\text { low tree-depth decom- } \\
\text { position }[84]\end{array}$ \\
\hline $\mathrm{p}$
\end{tabular}

Following [84], we will make use of the notation $\chi_{p}(G)$ for the minimum number of colors need for a vertex coloring of $G$ such that $i<p$ parts induce a subgraph of tree-depth at most $i$. These graph invariants ("generalized chromatc numbers") form a non-decreasing sequence:

$$
\chi(G)=\chi_{1}(G) \leq \chi_{2}(G) \leq \cdots \leq \chi_{p}(G) \leq \cdots \leq \chi_{\infty}(G)=\operatorname{td}(G) .
$$


Also, we say that a vertex coloring of a graph $G$ is a p-centered coloring if, for any (induced) connected subgraph $H$, either some color $c(H)$ appears exactly once in $H$, or $H$ gets at least $p$ colors.

The main result of [88] is the proof that these notions are related and that asking for the $\chi_{p}$ 's to be bounded on a class of graph (with bounds depending on $p$ ) is the same as requiring that the class has bounded expansion:

Theorem 4.3 ([88]). Let $\mathcal{C}$ be a class of graphs. The following conditions are equivalent:

(1) $\mathcal{C}$ has low tree-width colorings,

(2) $\mathcal{C}$ has low tree-depth colorings,

(3) for every integer $p,\left\{\chi_{p}(G): G \in \mathcal{C}\right\}$ is bounded,

(4) for every integer $p$, there exists an integer $X(p)$ such that every graph $G \in \mathcal{C}$ has a p-centered colorings using at most $X(p)$ colors,

(5) $\mathcal{C}$ has bounded expansion.

More precisely, the properties of having bounded $\chi_{p}$ and bounded $\nabla_{r}$ are related in [88] as shown Fig. 4.3:

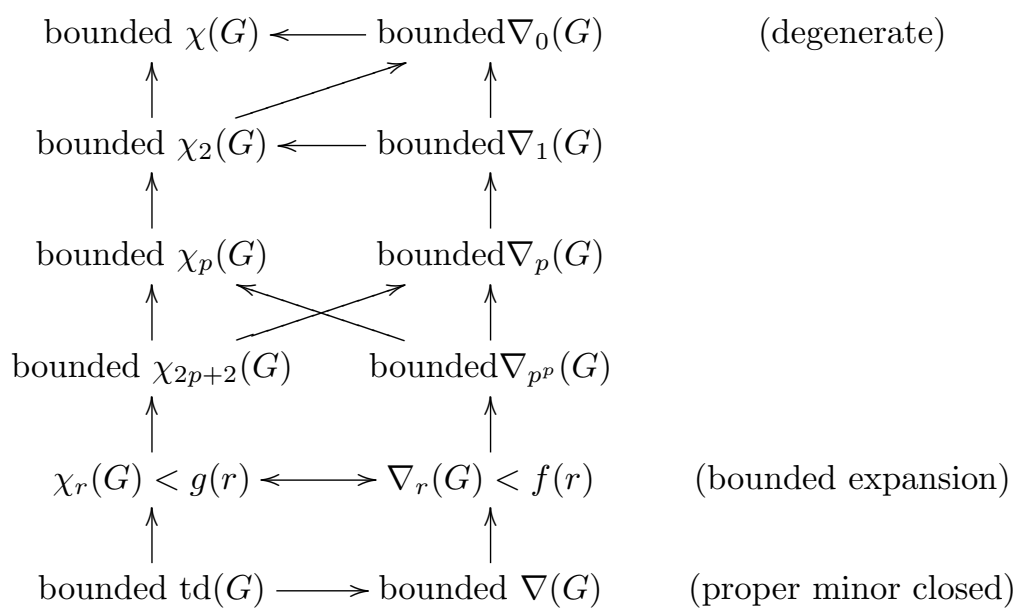

Figure 4.3. Invariant dependence

Further improvements have been obtained in bounding $\chi_{p}(G)$ in terms of the grads of $G$ [120] [38]. The best bound up to now is: 
Theorem $4.4([38])$. For each $p>0$, there exists a polynomial $P$ of degree $O\left(8^{p}\right)$ such that for each graph $G$,

$$
\chi_{p}(G) \leq P\left(\nabla_{2^{p-1}-1}(G)\right)
$$

As a consequence of these results (and of the above dependency schema), we also have the following equivalence:

Theorem 4.5 ([91]). Let $\mathcal{C}$ be an infinite (and not size bounded) class of graphs. The following conditions are equivalent:

- $\lim _{p \rightarrow \infty} \limsup _{G \in \mathcal{C}} \frac{\log \chi_{p}(G)}{\log |G|}=0$,

- $\lim _{r \rightarrow \infty} \limsup _{G \in \mathcal{C}} \frac{\log \nabla_{r}(G)}{\log |G|}=0$,

- $\mathcal{C}$ is a class of nowhere dense graphs.

Further characterizations are stated in the final Chapter.

\subsection{Algorithmic Considerations}

The decomposition algorithms we present here are those described in [83] [89]. They are based on indegree bounded orientations and transitive fraternal augmentations of these (see [88] for the relation between transitive fraternal augmentations and low tree-depth decompositions; see also [79] [82]).

Let $\vec{G}$ be a directed graph. A 1-transitive fraternal augmentation of $\vec{G}$ is a directed graph $\vec{H}$ with the same vertex set, including all the $\operatorname{arcs}$ of $\vec{G}$ and such that, for every vertices $x, y, z$,

- if $(x, z)$ and $(z, y)$ are $\operatorname{arcs}$ of $\vec{G}$ then $(x, y)$ is an arc of $\vec{H}$ (transitivity),

- if $(x, z)$ and $(y, z)$ are $\operatorname{arcs}$ of $\vec{G}$ then $(x, y)$ or $(y, x)$ is an arc of $\vec{H}$ (fraternity).

A 1-transitive fraternal augmentation $\vec{H}$ of $\vec{G}$ is tight if for each $\operatorname{arc}(x, y)$ in $\vec{H}$ which is not in $\vec{G}$ there exists a vertex $z$ so that $(x, z)$ and at least one of $(z, y),(y, z)$ are $\operatorname{arcs}$ of $\vec{G}$.

A transitive fraternal augmentation of a directed graph $\vec{G}$ is a sequence $\vec{G}=\vec{G}_{1} \subseteq \vec{G}_{2} \subseteq \cdots \subseteq \vec{G}_{i} \subseteq \vec{G}_{i+1} \subseteq \cdots$, such that $\vec{G}_{i+1}$ is a 1-transitive fraternal augmentation of $\vec{G}_{i}$ for every $i \geq 1$. The transitive fraternal augmentation is tight if all the 1-transitive fraternal augmentations of the sequence are tight. 


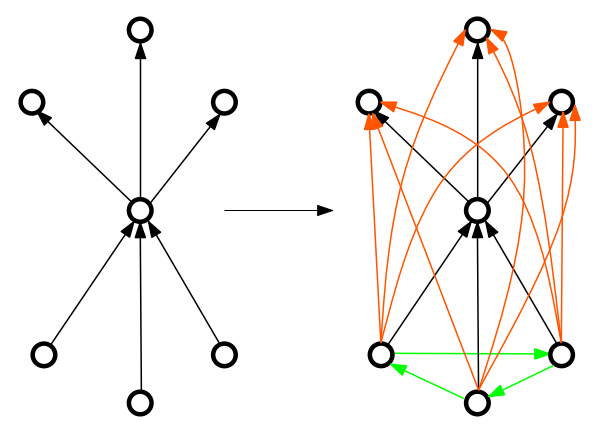

Figure 4.4. The Transitive Fraternal Augmentation of a Graph

4.6.1. Computing a Transitive Fraternal Augmentation. We describe an algorithm which computes, given any directed graph $\vec{G}$ of order $n$, a 1tight transitive fraternal augmentation $\vec{H}$ in $O\left(\Delta^{-}(\vec{G})^{2} n\right)$-time, such that:

$$
\begin{aligned}
\Delta^{-}(\vec{H}) & \leq f\left(\Delta^{-}\left(\vec{G}, \nabla_{1}(\vec{G})\right)\right. \\
\nabla_{r}(\vec{H}) & \leq g_{r}\left(\Delta^{-}(\vec{G}), \nabla_{2 r+1}(\vec{G})\right)
\end{aligned}
$$

for some polynomials $f$ and $g_{r}(r \in \mathbb{N})$.

In the augmentation process, we add two kind of arcs: transitivity arcs and fraternity arcs. Let us start with transitivity ones:

Require: $D$ represents the directed graph to be augmented.

Ensure: $D^{\prime}$ represents the array of the added arcs.

Initialize $D^{\prime}$.

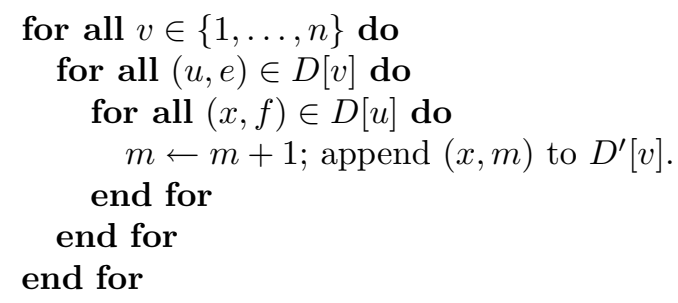

This algorithm runs in $O\left(\Delta^{-}(\vec{G})^{2} n\right)$ time, where $\Delta^{-}(\vec{G})$ is the maximum indegree of the graph to be augmented. It computes the list array $D^{\prime}$ of the 
transitivity arcs which are missing in $\vec{G}$, missing arcs may appear more than once in the list, but the number of added edges cannot exceed $\Delta^{-}(\vec{G})^{2} n$.

Now, we shall consider the fraternity edges.

Require: $D$ represents the directed graph to be augmented.

Ensure: $L$ represents the list of edges to be added.

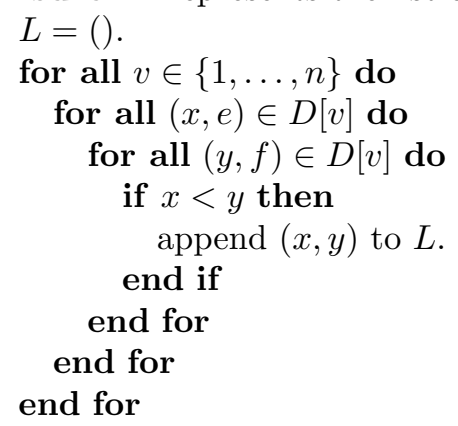

This algorithm runs in $O\left(\Delta^{-}(\vec{G})^{2} n\right)$-time and computes the list of the fraternity edges. Edges may appear in this list more than once but the length of the list $L$ cannot exceed $\Delta^{-}(\vec{G})^{2} n / 2$.

The simplification of $L$ (that is the removal of multiple instances of a same edge in the list), the computation of an acyclic orientation of the graph with edge set $L$ having minimum possible maximum indegree and the merge/simplification with the $\operatorname{arcs}$ in $D$ and $D^{\prime}$ may be achieved in $O\left(\Delta^{-}(\vec{G})^{2} n\right)$-time.

Let $G$ be a graph. Define $f(r)=\nabla_{r}(G)$ and $F(x, y)=x^{2}+2 y$ and let $R(p)=1+(p-1)\left(2+\left\lceil\log _{2} p\right\rceil\right)$. The tight fraternal augmentation $\vec{G}=$ $\vec{G}_{1} \subseteq \vec{G}_{2} \subseteq \cdots \subseteq \vec{G}_{R(p)}$ of $G$ computed by iterating $R(p)$ times the tight 1transitive fraternal augmentation algorithm is such that any proper coloring of $G_{R(p)}$ defines a $p$-centered coloring of $G$. Using the fact that a proper coloring of $G_{R(p)}$ using at most $\left\lfloor 2 \nabla_{0}\left(G_{R(p)}\right)\right\rfloor+1$ colors may easily been computed in $O(n)$-time, we get an algorithm which computes a $p$-centered coloring of $G$ using at most $C_{p}\left(\nabla_{p^{p}}(G)\right)$ colors in time $C_{p}^{\prime}\left(\nabla_{p^{p}}(G)\right) n$ where $C_{p}$ and $C_{p}^{\prime}$ are polynomials.

From this follows, in particular that for every fixed $p$, our $p$-centered coloring algorithm has the following properties: 


\begin{tabular}{|c|l|l|}
\hline \multicolumn{3}{|c|}{$p$-centered coloring characteristics } \\
\hline \multicolumn{1}{|c|}{ Class type } & Max number of color & Max running time \\
\hline \hline with bounded expansion & $O(1)$ & $O(n)$ \\
\hline of nowhere dense graphs & $n^{o(1)}$ & $\leq n^{1+o(1)}$ \\
\hline
\end{tabular}

\section{Algorithmic Applications}

In this Chapter we give a sample of algorithmic applicatons. Such applications seem to be typified by this situaton: Results which were proved earlier for planar graphs, and later sometimes generalized for proper minor closed classes can be sometimes proved for general classes with bounded expansion. And for bounded degree graphs we can sometimes proceede similarly. We review sample of such instances, for other applications see [83].

\subsection{SUbGraph IsOmorphism Problem}

Eppstein [44] gives a linear time algorithm to solve the subgraph isomorphism problem for a fixed pattern in a planar graph. He also gives a linear time bound for a fixed pattern and an input graph with bounded tree width decomposition. From this lemma and using our $p$-centered coloring algorithm, we deduce an extension of Eppstein's result of [44][45] to classes with bounded expansion:

Theorem 5.1. Let $\mathcal{C}$ be a class with bounded expansion and let $H$ be a fixed graph. Then there exists a linear time algorithm which computes, from a pair $(G, S)$ formed by a graph $G \in \mathcal{C}$ and a subset $S$ of vertices of $G$, the number of isomorphs of $H$ in $G$ that include some vertex in $S$. There also exists an algorithm running in time $O(n)+O(k)$ listing all such isomorphism where $k$ denotes the number of isomorphs (thus represents the output size).

It is also possible to extend this result to classes of nowhere dense graphs, with a complexity increasing from $O(n)$ to $n^{1+o(1)}$. All of these results are summarized in the following table: 


\begin{tabular}{|l|l|l|}
\hline \multicolumn{3}{|c|}{ Subgraph isomorphism problem } \\
\hline \multicolumn{1}{|c|}{ Context } & \multicolumn{1}{c|}{ Complexity } & \multicolumn{1}{c|}{ Reference(s) } \\
\hline \hline General & $O\left(n^{0.792|V(H)|}\right)$ & {$[92]$ using [24] } \\
\hline Bounded tree-width & $O(n)$ & {$[44]$ (also [25][26]) } \\
\hline Planar & $O(n)$ & {$[43][44]$} \\
\hline Bounded genus & $O(n)$ & {$[45]$} \\
\hline $\begin{array}{l}\text { Bounded expansion } \\
\text { (includes the three } \\
\text { previous classes) }\end{array}$ & $O(n)$ & {$[83][89]$} \\
\hline Nowhere dense & $\leq n^{1+o(1)}$ & {$[91]$} \\
\hline
\end{tabular}

\subsection{Small Distance Checking}

The following result is a weighted extension of the basic observation that bounded orientations allows $O(1)$-time checking of adjacency [22].

Theorem 5.2. For any class $\mathcal{C}$ with bounded expansion and for any integer $k$, there exists a linear time preprocessing algorithm so that for any preprocessed $G \in \mathcal{C}$ and any pair $\{x, y\}$ of vertices of $G$ the value $\min (k, \operatorname{dist}(x, y))$ may be computed in $O(1)$-time.

Also, this results may be extended to classes of nowhere dense graphs, using a preprocessing algorithm in $n^{1+o(1)}$-time allowing $\min (k, \operatorname{dist}(x, y))$ to be computed in $n^{o(1)}$-time.

\subsection{Existential First-order Properties}

Monadic second-order logic (MSOL) is an extension of first-order logic (FOL) that includes subsets of vertex sets (i.e. we expand our language by monadic predicates). The following theorem of Courcelle has been applied to solve many optimization problems.

Theorem 5.3 (Courcelle [25][26]). Let $\mathcal{K}$ be class of finite graphs $G=$ $\langle V, E, R\rangle$ represented as $\tau_{2}$-structures, that is: by two sorts of elements (vertices $V$ and edges $E$ ) and an incidence relation $R$. Let $\phi$ be a $\operatorname{MSOL}\left(\tau_{2}\right)$ 
sentence. If $\mathcal{K}$ has bounded tree width and $G \in \mathcal{K}$, then checking whether $G \vDash \phi$ can be done in linear time.

From this theorem and our results (especially low tree depth decomposition) we deduce (see e.g. [83]):

Theorem 5.4. Let $\mathcal{C}$ be a class with bounded expansion and let $p$ be a fixed integer. Let $\phi$ be an existential FOL $\left(\tau_{2}\right)$ sentence. Then there exists a linear time algorithms to check whether an input graph $G \in \mathcal{C}$ satisfies $\phi$ or not.

Thus for instance we have([83]):

Theorem 5.5. Let $\mathcal{K}$ be a class with bounded expansion and let $H$ be a fixed graph. Then, for each of the next properties there exists a linear time algorithm to decide whether a graph $G \in \mathcal{K}$ satisfies them:

- $H$ has a homomorphism to $G$,

- $H$ is a subgraph of $G$,

- $H$ is an induced subgraph of $G$.

\subsection{Dominating Sets}

The Dominating Set problem (DSP) is defined as follows.

Input: A graph $G=(V, E)$ and an integer parameter $k$.

Question: Does there exist a dominating set of size $k$ or less for $G$, i.e., a set $V^{\prime} \subseteq V$ with $\left|V^{\prime}\right| \leq k$ and such that for all $u \in V-V^{\prime}$ there is a $v$ in $V^{\prime}$ for which $u v \in E$ ?

This is a classic NP-complete problem [50] which is also apparently not fixed parameter tractable (with respect to the parameter $k$ ) because it is known to be $\mathrm{W}[2]$-complete in the $\mathrm{W}$-hierarchy of fixed parameter complexity theory [34]. In this theory, any graph problem for which there is an algorithm with time complexity $O\left(f(k) n^{\alpha}\right)$, for some problem parameter $k$, where $n$ is the number of nodes in the graph and where $\alpha$ is a constant independent of $k$ and $n$, is said to be fixed parameter tractable (fpt).

DSP is fixed parameter tractable with respect to, for example, tree-width [6] and tree decompositions are computable in linear time, for fixed treewidth [13]. DSP is similar in definition to the vertex cover problem (VCP), but they seem to differ considerably in their fixed parameter tractability properties. The Robertson-Seymour theory of graph minors [104] can be used to show that VCP is fixed parameter tractable because vertex cover is closed with respect to taking minors, and fpt algorithms have been described [34] for VCP. But DSP is not closed with respect to taking minors. 
DSP remains NP-complete when restricted to planar graphs [50]. Fellows and Downey [33, 34] gave a search tree algorithm for this problem which has time complexity $O\left(11^{k} n\right)$, when the input is restricted to planar graphs.

In [42] it is shown, using the search tree approach, that the dominating set problem is fixed parameter tractable for graphs of bounded genus, with time complexity of $O\left((4 g+40)^{k} n^{2}\right)$ for graphs of genus $g \geq 1$.

The idea to make this problem tractable, is to consider the strong properties of small dominating sets on classes with bounded tree-depth.

Let $G=(V, E)$ be a graph. A subset $X \subseteq V$ of $G$ is a dominating set of $G$ if every vertex of $G$ not in $X$ is adjacent to some vertex in $X$. We note $\mathfrak{D}(G)$ the family of all dominating sets of $G$ and by $\mathfrak{D}_{k}(G)$ the family of the dominating sets of $G$ having cardinality at most $k$.

For subsets $X, W \subseteq V$, we say that $X$ dominates $W$ if every vertex in $W \backslash X$ has a neighbor in $X$. We denote $\mathfrak{D}_{k}(G, W)$ the family of the subsets dominating $W$ and having cardinality at most $k$.

Lemma 5.6. For every integers $k, l \geq 1$ for every graph $G=(V, E)$ with tree-depth at most $l$ and for every subset $W \subseteq V$ of vertices, there exists a (blocker) subset $A=A(G, W) \subseteq V$ of at most $k l$ vertices meeting every $X \in \mathfrak{D}_{k}(G, W)$. Moreover, if a rooted forest $Y$ of height $l$ is given such that $G \subseteq \operatorname{clos}(Y)$ then we can find the blocker set $A$ in $O(k l)$-time.

From this Lemma, using a low tree-depth decomposition, we deduce:

Lemma 5.7. Let $\mathcal{C}$ be a class with bounded expansion. Then there exists a function $f: \mathbb{N} \rightarrow \mathbb{N}$ such that for every integer $k$, for every $G=(V, E) \in \mathcal{C}$ and for every $W \subseteq V$ a set $A(G, W)$ of cardinality at most $f(k)$ may be computed in $O(n)$-time (where $n$ is the order of $G$ ) which meets every set in $\mathfrak{D}_{k}(G, W)$.

Hence, by an easy induction on $k$ :

Theorem 5.8. Let $\mathcal{C}$ be a class with bounded expansion. Then there exists a function $g: \mathbb{N} \rightarrow \mathbb{N}$ such that for every integer $k$, every $G=(V, E) \in \mathcal{C}$ and every $W \subseteq V$ one may compute in time $O(g(k) n)$ a set $X$ which is either minimal set cardinality at most $k$ dominating $W$ or the empty set if $G$ has no dominating set of cardinality at most $k$.

Actually, we also deduce that any graph $G$ has at most $F\left(k, \nabla_{k^{k}}(G)\right)$ dominating sets of size at most $k$ and that they may be all enumerated in time $O\left(\phi\left(k, \nabla_{k^{k}}(G)\right) n\right)$. Notice that the result does not extend to the problem of finding a set $X$ of cardinality at most $k$ such that every vertex 
not in $X$ is at distance at most 2 from $X$ (consider $k$ disjoint stars of order $n / k$, giving $(n / k)^{k}$ possible solutions to the problem.

\subsection{InduCED MatChingS}

A matching in a graph $G$ is a subset of pairwise non-adjacent edges. An induced matching in a graph $G$ is a matching of $G$ which is an induced subgraph of $G$, that is a matching with the property that no endpoint of an edge in the matching is adjacent to an endpoint of another edge in the matching.

The problem of finding a maximum induced matching (that is: an induced matching with maximum cardinality) has been introduced by Stockmeyer and Vazirani [108] as the "risk-free marriage problem" and it was studied extensively $[37,46,48,53,107]$. For a graph $G$ we denote by $\beta^{*}(G)$ the size of a maximum induced matching.

It is known that the problem of deciding whether a given graph has an induced matching of size at least $k$ (for given $k$ ) is NP-complete [108], even for bipartite graphs of maximum degree 4. However, this problem has been shown to be solvable in polynomial time for several graph classes $[17,18,19,20,21,52,53,64,73,74]$ and even in linear time for trees $[49,53,122]$.

We consider here the approximation version of this problem. Given a NP-complete optimization problem $P$ (for example the computation of the size of a maximum induced matching), it is usual to look for an approximation algorithm $A_{P}$.If the ratio of the cost of a feasible solution computed by $A_{P}$ and the cost of an optimal solution is bounded by some constant then $R_{A_{P}}$ called the performance ratio of $A_{P}$. If $P$ admits an approximation algorithm with performance ratio $c$, then we say that $P$ is approximable within $c$. The class APX is the class of optimization problems that are approximable within $c$, for some constant $c$ [12]. The approximation problem associated to the maximum induced matching problem consists in looking for an induced matching the size of which is at least within a factor $c$ from the maximum. We say that $P$ admits a polynomial time approximation scheme (PTAS) if, given any $\epsilon>0$ there exists a polynomial-time approximation algorithm $A_{P, \epsilon}$ with performance ratio at most $1+\epsilon[76]$. An $A P X$-complete optimization problem is an optimization problem which belongs to APX and to which any APX problem has an L-reduction in polynomial time (see [99] and [11] for a formal definition of an L-reduction). An important property of APX-completeness is that an APX-complete optimization problem 
$Q$ does not admit a PTAS unless $\mathrm{P}=\mathrm{NP}$ [11]. In particular, there is some constant $c$ such that the problem of approximating $Q$ within $c$ is NP-hard.

Particularly, it is proved in [36] that in the class of $d$-regular graphs $(d \geq 3)$ the computation of $\beta^{*}(G)$ is approximable with asymptotic performance ratio $(d-1)$ (hence belongs to APX) but is APX-complete. The approximability is extended in [86] by proving that the problem of computing $\beta^{*}(G)$ is in APX when restricted to graphs with bounded $\nabla_{1}(G)$ (and thus to all bounded expansion classes).

A vertex $v$ of a graph $G$ is a clone if $G$ has a vertex $u \neq v$ with the same neighbourhood as $v$. In that say we say that $v$ is a clone of $u$. We denoted by $\sim$ be the equivalence relation defined by $x \sim y$ if $x$ and $y$ have the same neighbors (i.e. are clones). Let $G / \sim$ be the graph obtained by keeping exactly one vertex per equivalence class of $\sim$.

Theorem 5.9. Let $G$ be a connected graph. Then

$$
\frac{|V(G / \sim)|}{f\left(\nabla_{0}(G), \nabla_{1}(G)\right)} \leq \beta^{*}(G) \leq \frac{|V(G / \sim)|}{2}
$$

where

$$
f(x, y)=4 x\left(2^{2 y}+y+1\right)\left(2 x\left(2^{2 y}+y+1\right)+1\right)^{2}
$$

There is also an easy approximation algorithm: simply delete the clones in a graph.

Actually, a more general result is proved in [86]:

Theorem 5.10. For every integer $k>2$ and every $C>0$ there exists $\epsilon>0$ such that every connected graph $G$ of order $n$ with no involutive automorphism $\varphi$ exchanging two connected $P_{k}$-free subgraphs and such that $\nabla_{\lfloor k / 2\rfloor}(G)<C$ has a subset of $k \epsilon n$ vertices inducing $\epsilon n$ disjoint paths of order $k$.

\subsection{Vertex Separators}

A celebrated theorem of Lipton and Tarjan [71] states that any planar graph has a separator of size $O(\sqrt{n})$. Alon, Seymour and Thomas [4][3] showed that excluding $K_{h}$ as a minor ensures the existence of a separator of size at most $O\left(h^{3 / 2} \sqrt{n}\right)$. Gilbert, Hutchinson, and Tarjan [51] further proved that graphs with genus $g$ have a separator of size $O(\sqrt{g n})$ (this result is optimal). 
Plotkin et al. [100] introduced the concept of limited-depth minor exclusion and have shown that exclusion of small limited-depth minors implies the existence of a small separator. Precisely, they prove that any graph excluding $K_{h}$ as a depth $l$ minor has a separator of size $O\left(l h^{2} \log n+n / l\right)$ hence proving that excluding a $K_{h}$ minor ensures the existence of a separator of size $O(h \sqrt{n} \log n)$.

Plotkin et al. [100] proved that for graphs with $m$ edges and $n$ nodes, and integers $l$ and $h$, there is an $O(m n / l)$ time algorithm that will either produce a $K_{h}$-minor of depth at most $l \log n$ or will find a separator of size at most $O\left(n / l+4 l h^{2} \log n\right)$. We deduce that classes with sub-exponential expansions have separators of sub-linear size. Random cubic graphs having expansion bounded by $f(x)=2^{x}$ and almost surely $\Omega(n)$ bisection width [67] (thus $\Omega(n)$ separators) show that this result is optimal.

Theorem 5.11. Let $\mathcal{C}$ be a class of graphs with expansion bounded by a function $f$ such that $\log f(x)=o(x)$.

Then the graphs of order $n$ in $\mathcal{C}$ have separators of size $s(n)=o(n)$ which may be computed in time $O(n s(n))=o\left(n^{2}\right)$.

As random cubic graphs almost surely have bisection width at least $0.101 n$, they have almost surely no separator of size smaller than $n / 20$ It follows that if $\log f(x)=(\log 2) x$, the graphs have no sublinear separators any more. This shows the optimality of Theorem 5.11. More: as proved by Dvořák, the abscence of small vertex separators implies that the expansion of a class of graphs has to be sub-exponential. Precisely:

Theorem $5.12([38])$. If $\mathcal{C}$ is a monotone class of graphs such that each graph in $\mathcal{C}$ of order $n$ has a vertex separator of size $o\left(\frac{n}{\log n}\right)$, then $\mathcal{C}$ has subexponential expansion.

\section{Homomorphisms and Logic}

In this chapter we relate our theory to some problems treated in the context of model theory and mathematical logic: In Section 6.1 we deal with dualities and in Section 6.2 we deal with homomorphisms preservation theorems. Both these questions were intensively studied in the unrestricted cases $[97,105,7]$ as well as under various restrictions (to minor closed classes 
and classes of bounded degree graphs; see e.g. [8, 10, 27, 29, 35, 58, 84]. This research continued by considering classes of bounded expansion and, more recently, classes of nowhere dense graphs. A bit surprisingly, all the main results may be generalized by restriction to these classes.

This is proper place to say that the results of this section hold for more general structures than undirected graphs. They hold for oriented graphs, for colored graphs, hypergraphs and finite relational structures. It is easy to transform the results for graphs to results for hypergraphs and relational systems. This can be done using incidence graphs and, in most cases alternatively, using 2-sections (known in model theory as Gaifman graphs), see [91]. However to keep the style of this paper uniform we state most of the results for graphs only.

\subsection{Restricted Dualities}

Recall that a homomorphism from a graph $G$ to a graph $H$ is a mapping $f: V \rightarrow V(H)$ which preserves adjacency: $\{f(x), f(y)\} \in E(H)$ whenever $\{x, y\} \in E(G)$. We denote by $G \stackrel{f}{\longrightarrow} H$ or $f: G \longrightarrow H$ that $f$ is a homomorphism from $G$ to $H$. The existence of a homomorphism from $G$ to $H$ is denoted by $G \longrightarrow H$, while the non-existence of such a homomorphism is denoted by $G \longrightarrow H$. Graphs $G, G^{\prime}$ are said to be homomorphism equivalent if we have both $G \longrightarrow G^{\prime}$ and $G^{\prime} \longrightarrow G$. It is also clear that the relation $G \leq H$ defined as $G \longrightarrow H$ is a quasiorder on the class of all finite graphs. This quasiorder becomes a partial order if we restrict it to the class of all non-isomorphic minimal retracts (i.e. cores). This partial order is called the homomorphism order. All graphs considered in this paper are finite. A class is a (possibly infinite) class of finite graphs. See [60] for a recent introduction to graphs and homomorphisms.

The following definition is the central definition of this section:

Definition 6.1. A class of graphs $\mathcal{K}$ has all restricted dualities if, for any finite set of connected graphs $\mathcal{F}=\left\{F_{1}, F_{2}, \ldots, F_{t}\right\}$, there exists a set of finite graphs $\mathcal{D}_{\mathcal{F}}^{\mathcal{K}}$ such that $F_{i} \longrightarrow D$ for $i=1, \ldots, t$ and every $D \in \mathcal{D}_{\mathcal{F}}^{\mathcal{K}}$, and such that for all $G \in \mathcal{K}$,

$$
\left(F_{i} \longrightarrow G \text { for all } i=1,2, \ldots, t\right) \Longleftrightarrow\left(G \longrightarrow D \text { for some } D \in D_{\mathcal{F}}^{\mathcal{K}}\right) \text {. }
$$

Any instance of (3) is called a restricted finite duality (for the class $\mathcal{K}$ ), or $\mathcal{K}$-restricted duality. 
In the extremal case that $\mathcal{F}$ and $\mathcal{D}_{\mathcal{F}}^{\mathcal{K}}$ consists from single element sets we speak about restricted singleton duality (this case is however the key to the general case). Also note that if all graphs are connected then the set $\mathcal{D}_{\mathcal{F}}^{\mathcal{K}}$ can be chosen with one element.

We now justify this general definition by the following two examples and by the context in which this definition crystallized.

Example 1. The Grötzsch's celebrated theorem (see e.g. [115]) says that every triangle-free planar graph is 3 -colorable. In the language of homomorphisms this says that for every triangle-free planar graph $G$ there is a homomorphism of $G$ into $K_{3}$. Using the partial order terminology, Grötzsch's theorem says that $K_{3}$ is an upper bound (in the homomorphism order) for the class $\mathcal{P}_{3}$ of all planar triangle-free graphs. It is $K_{3} \notin \mathcal{P}_{3}$ and this suggests a natural question (first formulated in [78]): Is there yet a smaller bound? The answer, which may be viewed as a strengthening of Grötzsch's theorem, is positive: there exists a triangle free 3-colorable graph $H$ such that $G \longrightarrow H$ for every graph $G \in \mathcal{P}_{3}$. This has been proved in $[84,82]$ in a stronger form for all proper minor-closed classes. (The case of trianglefree planar graphs is interesting in its own and it has been related to a conjecture by Seymour and to Guenin's theorem [56] (see [77]) and seems to find a proper setting in the context of $T T$-continuous mappings, [94].) One can view these results as restricted dualities (which hold in the class of planar graphs). Restricted duality results have since been generalized not only to proper minor closed classes of graphs and but also to other forbidden subgraphs, in fact to any finite set of connected graphs, [84]. This then implies that Grötzsch's theorem can be strengthened by a sequence of even stronger bounds and that the supremum (in the homomorphism order) of the class of all triangle free planar graphs does not exist, [81].

Example 2. A graph is sub-cubic if the degrees of all its vertices are $\leq 3$. By Brooks theorem (see e.g. [32]) every sub-cubic connected graph is 3colorable with the single exception of $K_{4}$. What about the class of all sub-cubic triangle-free graphs? Does there exists a triangle free 3-colorable bound? The positive answer to this question is given in [35] and [58]. In fact for every finite set $\mathcal{F}=\left\{F_{1}, F_{2}, \ldots, F_{t}\right\}$ of connected graphs there exists a graph $H$ with the following property:

$G \longrightarrow H$ for every sub-cubic graph $G \in \operatorname{Forb}_{\mathrm{h}}(\mathcal{F})$. 
(Here $\operatorname{Forb}_{\mathrm{h}}(\mathcal{F})$ is the class of all graphs $G$ which satisfy $F_{i} \longrightarrow G$ for every $i=1,2, \ldots, t$. Thus $\operatorname{Forb}_{\mathrm{h}}\left(K_{3}\right)$ is the class of all triangle free graphs.) It follows that the class of all sub-cubic graphs has all restricted dualities.

Note that while sub-cubic graphs, and more generally graphs with bounded degrees, have all restricted dualities, this is not true for classes of degenerate graphs $[78,81]$.

Where lies the boundary for validity of restricted dualities? This is the central question of this area. We give a very general sufficient condition for a class to have all restricted dualities. To motivate these results we first introduce the original context of (unrestricted) dualities.

The following is a partial order formulation of an important homomorphism (or coloring; or Constraint Satisfaction) problem (this time we formulate the definition for finite structures):

Definition 6.2. A pair $F, D$ of structures is called a dual pair if for every structure $G$,

$$
F \longrightarrow G \Longleftrightarrow G \longrightarrow D .
$$

We also say that $F$ and $D$ form a duality, $D$ is called (singleton) dual of $F$. Dual pairs of graphs and of finite relational structures were characterized in [97], the notion itself goes back to [93]. Equivalently, one can describe a dual pair $F, D$ by saying that the structure $D$ is the maximum graph in the class $\operatorname{Forb}_{\mathrm{h}}(F)$ (maximum in the homomorphism order).

It appears (and this is the main result of [97]) that (up to homomorphism equivalence) all the dualities are of the form $\left(T, D_{T}\right)$ where $T$ is a finite (relational) tree. Every dual $D_{T}$ is uniquely determined (up to homomorphism equivalence) by the tree $T$ (but its structure is far more difficult to describe, see e.g. $[98,96,69])$. These results imply infinitely many examples of dualities. But a much richer spectrum (and in fact a surprising richness of results) is obtained by restricting the validity of (4) to a particular class of graphs $\mathcal{K}$. This then is expressed by the notion of a restricted duality.

It is easy to see that using the homomorphism order we can reformulate the restricted duality as follows: A class $\mathcal{K}$ has all restricted dualities if for any finite set of connected graphs $\mathcal{F}=\left\{F_{1}, F_{2}, \ldots, F_{t}\right\}$ the $\operatorname{class}^{\operatorname{Forb}_{\mathrm{h}}}(\mathcal{F}) \cap$ $\mathcal{K}$ has an upper bound in the homomorphism order (namely $D_{\mathcal{F}}^{\mathcal{K}}$ ) which belongs to the class $\operatorname{Forb}_{\mathrm{h}}(\mathcal{F})$.

Bounded expansion classes of graphs and structures provide a rich spectrum of restricted dualities. This has been shown in [88, 89, 90, 83]. The following may be see as one of the main results: 
Theorem 6.1. Any class of graphs (and more generally structures) with bounded expansion has all restricted dualities.

As both proper minor closed classes and bounded degree graphs form classes of bounded expansion this result generalizes both Examples 1 and 2. In fact the seeming incomparability of bounded degree graphs and minor closed classes led us to the definition of bounded expansion classes.

\subsection{Homomorphism Preservation}

Homomorphisms are one of the key concept of model theory as they are naturally related to the satisfiability of formulas. This we shall illustrate on homomorphism preservation theorems. This application provided the motivation for the concept of nowhere dense graphs and structures.

Classical model theory studies properties of abstract mathematical structures (finite or not) expressible in first-order logic, see e.g. [61]. In this context, three classical fundamental preservation theorems have been proved, which connect syntactic and semantic properties of first-order formulas:

- the Loś-Tarski theorem, which asserts that a first-order formula is preserved under extensions on all structures if, and only if, it is logically equivalent to an existential formula;

- Lyndon's theorem, which asserts that a first-order formula is preserved under surjective homomorphisms on all structures if, and only if, it is logically equivalent to a positive formula;

- the homomorphism preservation theorem which asserts that a firstorder formula is preserved under homomorphisms on all structures if, and only if, it is logically equivalent to an existential-positive formula.

The terms "all structures", which means finite and infinite structures, is crucial in the statement of these theorems.

Finite model theory is the study of the first-order logic (and its various extensions) on finite structures [40], [70]. In this context, it has been proved that the two first theorems fail when relativized to the finite, that is: there exists a first-order formula that is preserved under extensions on finite structures, but is not equivalent in the finite to an existential formula [112][57][2] and there exists a first-order formula that is preserved under surjective homomorphisms on finite structures, but is not equivalent in the finite to a positive formula [1][109]. However, a bit surprisingly, the relativized version of the homomorphism preservation theorem to the finite has been recently proved by B. Rossman [105]. 
Relativizations of homomorphism preservation theorem to specific classes of structures have been studied and in this context e.g. in $[9,8,10]$ and in this context A. Dawar defined classes of graphs called wide, almost wide and quasi-wide (see e.g. [27]) and thay were introduced in Section 2.3. Here we treat these interesting classes in a greater detail.

For instance, it has been proved in [8] that the extension preservation theorem holds in any class $\mathcal{C}$ that is wide, hereditary (i.e. closed under taking substructures) and closed under disjoint unions. Wide classes includes classes with bounded maximum degree. We prove here (and see [87] that an hereditary class of graphs is actually wide if and only if it has a bounded degree (Theorem 6.2).

Also, it has been proved in [9] [10] that the homomorphism preservation theorem holds in any class $\mathcal{C}$ that is almost wide, hereditary and closed under disjoint unions. Almost wide classes of graphs include classes of graphs which exclude a minor [68].

Dawar and Malod [28] recently announced that the homomorphism preservation theorem holds in any hereditary quasi-wide class that is closed under disjoint unions. This is a strengthening of the result proved in [9]. Clearly, quasi-wide quasi-wide classes of graphs include classes of graphs locally excluding a minor [29]. Using the theory developed for classes of sparse graphs we shall give a complete characterization of hereditary classes of graphs which are wide, almost wide and quasi-wide. In fact this led us to the definition of classes of nowhere dense structures.

We find it useful to study wide (and almost wide and quasi-wide) classes (defined already in Section 2.3) by means of the following functions $\Phi_{\mathcal{C}}$ and $\bar{\Phi}_{\mathcal{C}}$ defined for classes of graphs. It is essential for our approach that we also define the uniform version of these concepts.

Function $\Phi_{\mathcal{C}}$ This function has domain $\mathbb{N}$ and range $\mathbb{N} \cup\{\infty\}$ and $\Phi_{\mathcal{C}}(d)$ is defined for $d \geq 1$ as the minimum $s$ such that the class $\mathcal{C}$ satisfies the following property:

"There exists a function $F: \mathbb{N} \rightarrow \mathbb{N}$ such that for every integer $m$, every graph $G \in \mathcal{C}$ with order at least $F(m)$ contains a subset $S$ of size at most $s$ so that $G-S$ has a $d$-independent set of size $m$."

We put $\Phi_{\mathcal{C}}(d)=\infty$ if $\mathcal{C}$ does not satisfy the above property for any value of $s$ ). Moreover, we define $\Phi_{\mathcal{C}}(0)=0$.

Function $\bar{\Phi}_{\mathcal{C}}$ This function has domain $\mathbb{N}$ and range $\mathbb{N} \cup\{\infty\}$ and $\bar{\Phi}_{\mathcal{C}}(d)$ is defined for $d \geq 1$ as the minimum $s$ such that $\mathcal{C}$ satisfies the following property: 
"There exists a function $F: \mathbb{N} \rightarrow \mathbb{N}$ such that for every integer $m$, every graph $G \in \mathcal{C}$ and every subset $A$ of vertices of $G$ of size at least $F(m)$, the graph $G$ contains a subset $S$ of size at most $s$ so that $A$ includes a $d$-independent set of size $m$ of $G-S$."

We put $\bar{\Phi}_{\mathcal{C}}(d)=\infty$ if $\mathcal{C}$ does not satisfy the above property for any value of $s$ ). Moreover, we define $\bar{\Phi}_{\mathcal{C}}(0)=0$.

Notice that obviously $\bar{\Phi}_{\mathcal{C}} \geq \Phi_{\mathcal{C}}$ for every class $\mathcal{C}$ and for every integer $d$.

Using these functions we can formulate notions of wide, almost wide and quasi-wide classes (which were defined in Section 3.3) as follows:

A class of graphs $\mathcal{C}$ is wide (resp. almost wide, resp. quasi-wide) if $\Phi_{\mathcal{C}}$ is identically 0 (resp. bounded, resp. finite) [27]:

$$
\begin{aligned}
\mathcal{C} \text { is wide } & \Longleftrightarrow \forall d \in \mathbb{N}: \Phi_{\mathcal{C}}(d)=0 \\
\mathcal{C} \text { is almost wide } & \Longleftrightarrow \sup _{d \in \mathbb{N}} \Phi_{\mathcal{C}}(d)<\infty \\
\mathcal{C} \text { is quasi-wide } & \Longleftrightarrow \quad \forall d \in \mathbb{N}: \Phi_{\mathcal{C}}(d)<\infty
\end{aligned}
$$

Notice that a hereditary class $\mathcal{C}$ is wide (resp. almost wide, resp. quasiwide) if and only if $\mathcal{C} \nabla 0$ is wide (resp. almost wide, resp. quasi-wide) as deleting edges cannot make it more difficult to find independent sets.

We introduce the following variation of the above definitions: A class of graphs $\mathcal{C}$ is uniformly wide (resp. uniformly almost wide, resp. uniformly quasi-wide) if $\bar{\Phi}_{\mathcal{C}}$ is identically 0 (resp. bounded, resp. finite):

$$
\mathcal{C} \text { is uniformly wide } \quad \Longleftrightarrow \quad \forall d \in \mathbb{N}: \bar{\Phi}_{\mathcal{C}}(d)=0
$$

$\mathcal{C}$ is uniformly almost wide $\Longleftrightarrow \sup _{d \in \mathbb{N}} \bar{\Phi}_{\mathcal{C}}(d)<\infty$

$$
\mathcal{C} \text { is uniformly quasi-wide } \quad \Longleftrightarrow \quad \forall d \in \mathbb{N}: \bar{\Phi}_{\mathcal{C}}(d)<\infty
$$

Notice that a class $\mathcal{C}$ is uniformly wide (resp. uniformly almost wide, resp. uniformly quasi-wide) if and only if $\mathcal{C} \nabla 0$ is uniformly wide (resp. uniformly almost wide, resp. uniformly quasi-wide) as the property is hereditary in nature and deleting edges cannot make it more difficult to find independent sets.

Theorem $6.2([87])$. Let $\mathcal{C}$ be a hereditary class of graphs. Then the following are equivalent:

- $\Delta(\mathcal{C})<\infty$,

- $\mathcal{C}$ is wide,

- $\mathcal{C}$ is uniformly wide. 
Before characterizing almost wide classes we state a quantitative result relating these classes to bounded expansion.

Theorem 6.3 ([87]). Let $\mathcal{C}$ be a class with bounded expansion. Then $\bar{\Phi}_{d}(\mathcal{C}) \leq \nabla_{\lfloor d / 2\rfloor-1}(\mathcal{C})$.

As a consequence we have the following characterization of hereditary almost wide classes of graphs, which gives a positive answer to a question of Dawar whether classes of graphs more general than those excluding a minor could be proved to be almost wide [27].

Theorem 6.4 ([87]). Let $\mathcal{C}$ be a hereditary class of graphs. Then the following are equivalent:

- $\mathcal{C}$ is almost wide;

- $\mathcal{C}$ is uniformly almost wide;

- There are $s \in \mathbb{N}$ and $t: \mathbb{N} \rightarrow \mathbb{N}$ such that $K_{s, t(r)} \notin \mathcal{C} \nabla r$ (for all $r \in \mathbb{N}$ ).

If $\mathcal{C}$ is actually minor closed class the we can be more precise:

Theorem $6.5([87])$. Let $\mathcal{C}$ be a minor closed class of graphs and let $s$ be an integer. Then the following are equivalent:

- $\mathcal{C}$ is almost wide and $\Phi_{\mathcal{C}}(d)<s$ for every integer $d \geq 2$;

- $\mathcal{C}$ is uniformly almost wide and $\bar{\Phi}_{\mathcal{C}}(d)<s$ for every integer $d \geq 2$;

- $\mathcal{C}$ excludes some graph $K_{s, t}$.

For instance, consider a surface $\Sigma$ and let $\mathcal{C}_{\Sigma}$ be the class of the graphs which embed on $\Sigma$. It has been proved in [10] that $\mathcal{C}_{\Sigma}$ is almost wide (for every surface $\Sigma$ ) and that $\Phi_{\mathcal{C}_{\Sigma}}(d)$ is at most equal to the order of the smallest clique which does not embed on $\Sigma$. Actually, $\Phi_{\mathcal{C}_{\Sigma}}(d)=\bar{\Phi}_{\mathcal{C}_{\Sigma}}(d)=2$ for every integer $d$, as every $K_{2, n}$ embed on any surface but not every $K_{3, n}$ does.

Finally, we have the following characterization of quasi-wide classes:

Theorem 6.6 ([87]). Let $\mathcal{C}$ be a hereditary class of graphs. The following conditions are equivalent:

- $\mathcal{C}$ is quasi-wide;

- $\mathcal{C}$ is uniformly quasi-wide;

- for every integer $d$ there is an integer $N$ such that $K_{N} \notin \mathcal{C} \nabla d$;

- $\mathcal{C}$ is a class of nowhere dense graphs.

This then implies (using the above mentioned result of Dawar and Molod [28]) that the relativized homomorphism preservation theorem holds for all classes of nowhere dense graphs. Perhaps these result indicate that classes 
with bounded expansion and classes of nowhere dense graphs provide a proper setting for this type of questions (about wide, semi-wide and quasiwide classes) and we obtain characterization theorems (which are reviewed in the last chapter).

\subsection{Richness of First Order}

A class $\mathcal{K}$ is said to be first order definable if there exists a first order formula $\Phi$ such that $\mathcal{K}$ is the class of all structures which are models of $\Phi$. This can be obviously relativized: A subclass $\mathcal{L}$ of $\mathcal{K}$ is said to be first order definable in the class $\mathcal{K}$ if $\mathcal{L}$ is just the class of all structures in $\mathcal{K}$ which model $\Phi$. However, if a class $\mathcal{L}$ is defined by an existentially positive first order formula then $\mathcal{L}$ is defined as the class of all structures in $\mathcal{K}$ for which there exist a homomorphism from a finite set $\mathcal{F}$ of structures. This in turn means that the complementary class $\mathcal{L}^{\prime}$ of $\mathcal{L}$ is the class of all structures $\mathbf{A}$ for which there is no homomorphism $\mathbf{F} \longrightarrow \mathbf{A}$ for any $\mathbf{F} \in \mathcal{F}$. In the other words the complementary class is the class $\operatorname{Forb}_{\mathrm{h}}(\mathcal{F})$. This setting is close to (homomorphism ) dualities and to homomorphism preservation theorems.

Combining the above Theorems $6.3,6.1$ we obtain the following:

Theorem 6.7. Let $\mathcal{K}$ be a bounded expansion class of structures. For a homomorphism closed subclass $\mathcal{L}$ of $\mathcal{K}$ are the following statements equivalent:

- $\mathcal{L}$ is first order definable in $\mathcal{K}$;

- $\mathcal{L}^{\prime}=\operatorname{Forb}_{\mathrm{h}}(\mathcal{F})$ for a finite set $\mathcal{F}$ of structures;

- $\mathcal{L}$ is defined by a (finite) $\mathcal{K}$-restricted duality.

Combining with the results of [90] we prove perhaps surprising fact that any homomorphism closed first order property when restricted to a class with bounded expansion is a restricted finite duality. Thus we obtained the restricted analogy of the following first order characterization of (unrestricted) dualities:

Theorem 6.8. For a homomorphism closed subclass $\mathcal{L}$ of graphs (structures) are the following statements equivalent:

- $\mathcal{L}$ is first order definable;

- $\mathcal{L}^{\prime}=\operatorname{Forb}_{\mathrm{h}}(\mathcal{F})$ for a finite set $\mathcal{F}$ of structures;

- $\mathcal{L}$ is defined by a (finite) duality.

This is a combinatorion of [97] and [7] (and also [105]). 


\section{Summary (Characterization Theorems)}

\subsection{Polynomial Dependence}

Two graph invariants $f$ and $g$ are polynomially dependent, and we note $f \asymp g$, if there exists polynomials $P, Q$ such that for every graph $G$ :

$$
f(G) \leq P(g(G)) \quad \text { and } \quad g(G) \leq Q(f(G)) .
$$

Notice that $f \asymp g$ is equivalent to $\log f=\Theta(\log g)$.

For instance we have seen above in Section 2.3 and in Section 1.3 that

$$
\begin{aligned}
\operatorname{col}_{p} & \asymp \operatorname{wcol}_{p}, \\
\nabla_{r} & \asymp \widetilde{\nabla}_{r} .
\end{aligned}
$$

Also, we may extend this property to functions of more than one graph and express concisely the main result of Section 1.4:

$$
\nabla_{r}(G \bullet H) \asymp \nabla_{r}(G)|H| .
$$

(This is a direct consequence of Lemma 2.4 and Lema 2.5.)

We also consider a weaker form of dependence for invariant sequences: $\left(f_{i}\right)_{i \in \mathbb{N}}$ and $\left(g_{i}\right)_{i \in \mathbb{N}}$ which are said to be weakly polynomially dependent, and we note $\left(f_{i}\right)_{i \in \mathbb{N}} \stackrel{\star}{=}\left(g_{i}\right)_{i \in \mathbb{N}}$ if there exists $\alpha, \beta: \mathbb{N} \rightarrow \mathbb{N}$ and polynomials $\left(P_{i}\right)_{i \in \mathbb{N}},\left(Q_{i}\right)_{i \in \mathbb{N}}$ such that for every integer $i$ and every graph $G$ :

$$
f_{i}(G) \leq P_{i}\left(g_{\alpha(i)}(G)\right) \quad \text { and } \quad g_{i}(G) \leq Q_{i}\left(f_{\beta(i)}(G)\right) .
$$

In this notation we have for instance:

$$
\left(\chi_{i}\right)_{i \in \mathbb{N}} \stackrel{\star}{\asymp}\left(\nabla_{i}\right)_{i \in \mathbb{N}} \stackrel{\star}{\longleftarrow}\left(\mathrm{wcol}_{i}\right)_{i \in \mathbb{N}}
$$

and

$$
\left(\omega_{i}\right)_{i \in \mathbb{N}} \stackrel{\star}{\asymp}\left(\widetilde{\omega}_{i}\right)_{i \in \mathbb{N}},
$$

where $\omega_{i}(G)=\omega(G \nabla i)$ and $\widetilde{\omega}_{i}(G)=\omega(G \widetilde{\nabla} i)$.

\subsection{Characterizations}

In this section, we state some characterizations of sparse classes, which are mainly consequences of two aspects:

- the polynomial dependence (and weak polynomial dependence) of certain graph invariants, like $\nabla_{r}, \widetilde{\nabla}_{r}, \chi_{p}, \operatorname{col}_{p}, \operatorname{wcol}_{p}$, etc.

- the characterization of uniformly quasi-wide classes. 
These three characterization theorems perhaps present a fitting conclusion for this survey.

\subsubsection{Classes of Nowhere Dense Graphs.}

Theorem 7.1 ([91] and [87]). Let $\mathcal{C}$ be an unbounded size infinite class of graphs. Then the following conditions are equivalent:

(1) $\mathcal{C}$ is a class of nowhere dense graphs,

(2) for every integer $r, \mathcal{C} \nabla r$ is not the class of all finite graphs,

(3) for every integer $r, \mathcal{C} \widetilde{\nabla} r$ is not the class of all finite graphs,

(4) $\mathcal{C}$ is a uniformly quasi-wide class,

(5) $H(\mathcal{C})$ is a quasi-wide class,

(6) $\lim _{r \rightarrow \infty} \limsup _{G \in \mathcal{C} \nabla r} \frac{\log \|G\|}{\log |G|}=1$,

(7) $\lim _{r \rightarrow \infty} \limsup _{G \in \mathcal{C} \widetilde{\nabla} r} \frac{\log \|G\|}{\log |G|}=1$,

(8) $\lim _{r \rightarrow \infty} \limsup _{G \in \mathcal{C}} \frac{\log \nabla_{r}(G)}{\log |G|}=0$,

(9) $\lim _{r \rightarrow \infty} \limsup _{G \in \mathcal{C}} \frac{\log \widetilde{\nabla}_{r}(G)}{\log |G|}=0$,

(10) $\lim _{p \rightarrow \infty} \limsup _{G \in \mathcal{C}} \frac{\log \chi_{p}(G)}{\log |G|}=0$,

(11) $\lim _{p \rightarrow \infty} \limsup _{G \in \mathcal{C}} \frac{\log \operatorname{col}_{p}(G)}{\log |G|}=0$,

(12) $\lim _{p \rightarrow \infty} \limsup _{G \in \mathcal{C}} \frac{\log \mathrm{wcol}_{p}(G)}{\log |G|}=0$,

(13) for every integer $c$, the class $\mathcal{C} \bullet K_{c}=\left\{G \bullet K_{c}: G \in \mathcal{C}\right\}$ is a class of nowhere dense graphs,

(14) for every integer $p$, every graph $G \in \mathcal{C}$ has a p-centered colorings using at most $|G|^{o(1)}$ colors,

(15) for every polynomial $P$, the class $\mathcal{C}^{\prime}$ of the 1-transitive fraternal augmentations of directed graphs $\vec{G}$ with $\Delta^{-}(\vec{G}) \leq P\left(\nabla_{0}(G)\right)$ and $G \in \mathcal{C}$ form a class of nowhere dense graphs,

\subsubsection{Bounded Expansion Classes.}

Theorem 7.2 ([88] and [120]). Let $\mathcal{C}$ be a class of graphs. Then the following conditions are equivalent:

(1) $\mathcal{C}$ has bounded expansion,

(2) for every integer $r, \sup _{G \in \mathcal{C}} \nabla_{r}(G)<\infty$, 
(3) for every integer $r, \sup _{G \in \mathcal{C}} \widetilde{\nabla}_{r}(G)<\infty$,

(4) for every integer $p, \sup _{G \in \mathcal{C}} \chi_{p}(G)<\infty$,

(5) for every integer $p, \sup _{G \in \mathcal{C}} \operatorname{col}_{p}(G)<\infty$,

(6) for every integer $p, \sup _{G \in \mathcal{C}} \operatorname{wcol}_{p}(G)<\infty$,

(7) for every integer $c$, the class $\mathcal{C} \bullet K_{c}=\left\{G \bullet K_{c}: G \in \mathcal{C}\right\}$ has bounded expansion,

(8) $\mathcal{C}$ has low tree-width colorings,

(9) $\mathcal{C}$ has low tree-depth colorings,

(10) for every integer $p$, there exists an integer $X(p)$ such that every graph $G \in \mathcal{C}$ has a p-centered colorings using at most $X(p)$ colors,

(11) for every integer $k$, the class $\mathcal{C}^{\prime}$ of the 1-transitive fraternal augmentations of directed graphs $\vec{G}$ with $\Delta^{-}(\vec{G}) \leq k$ and $G \in \mathcal{C}$ form a class with bounded expansion,

$(12)$ the class $\mathcal{C}$ is a degenerate class of graphs (that is: $\nabla_{0}(G)$ is bounded on $\mathcal{C})$ and there exists a function $F$ such that every orientation $\vec{G}$ of a graph $G \in \mathcal{C}$ has a transitive fraternal augmentation $\vec{G}=\vec{G}_{1} \subseteq$ $\vec{G}_{2} \subseteq \cdots \subseteq \vec{G}_{i} \subseteq \cdots$ where $\Delta^{-}\left(\vec{G}_{i}\right) \leq Q\left(\Delta^{-}(\vec{G}), i\right)$,

(13) there exists a function $f$ such that every graph $G \in \mathcal{C}$ has a transitive fraternal augmentation $\vec{G}=\vec{G}_{1} \subseteq \vec{G}_{2} \subseteq \cdots \subseteq \vec{G}_{i} \subseteq \cdots$ where $\Delta^{-}\left(\vec{G}_{i}\right) \leq f(i)$.

\subsubsection{Bounded Tree-depth Classes.}

Theorem 7.3. Let $\mathcal{C}$ be a class of graphs. The following conditions are equivalent:

(1) $\mathcal{C}$ has bounded tree-depth,

(2) there exists an integer $l(\mathcal{C})$ such that no graph $G \in \mathcal{C}$ includes a path of length greater than $l(\mathcal{C})$,

(3) $\mathcal{C}$ is degenerate (i.e. $\nabla_{0}(\mathcal{C})<\infty$ ) and there exists an integer $L(\mathcal{C})$ such that no graph $G \in \mathcal{C}$ includes an induced path of length greater than $L(\mathcal{C})$,

(4) $\lim _{p \rightarrow \infty} \chi_{p}(\mathcal{C})<\infty$,

(5) $\lim _{p \rightarrow \infty} \operatorname{col}_{p}(\mathcal{C})<\infty$,

(6) $\lim _{p \rightarrow \infty} \operatorname{wcol}_{p}(\mathcal{C})<\infty$

\section{REFERENCES}

[1] M. Ajtai and Y. Gurevich, Monotone versus positive, Journal of the ACM 34 (1987), 1004-1015. 
[2] N. Alechina and Y. Gurevich, Syntax vs. semantics on nite structures, Structures in Logic and Computer Science (1997), 14-33.

[3] N. Alon, P.D. Seymour, and R. Thomas, A separator theorem for graphs with excluded minor and its applications, Proceedings of the 22nd Annual ACM Symposium on Theory of Computing, 1990, pp. 293-299.

[4] _ A separator theorem for nonplanar graphs, Journal of the American Mathematical Society 3 (1990), 801-808.

[5] N. Alon and A. Shapira, A characterization of the (natural) graph properties testable with one-sided error, Proc. 46th IEEE FOCS, 2005, pp. 429-438.

[6] S. Arnborg and A. Proskurowski, Linear time algorithms for NP-hard problems restricted to partial k-trees, Discrete Applied Mathematics 23 (1989), no. 1, 11-24.

[7] A. Atserias, On digraph coloring problems and treewidth duality, 20th IEEE Symposium on Logic in Computer Science (LICS), 2005, pp. 106-115.

[8] A. Atserias, A. Dawar, and M. Grohe, Preservation under extensions on wellbehaved finite structures, 32nd International Colloquium on Automata, Languages and Programming (ICALP) (Springer-Verlag, ed.), Lecture Notes in Computer Science, vol. 3580, 2005, pp. 1437-1449.

[9] A. Atserias, A. Dawar, and P.G. Kolaitis, On preservation under homomorphisms and unions of conjunctive queries, Proceedings of the twenty-third ACM SIGMODSIGACT-SIGART symposium on Principles of database systems, ACM Press, 2004, pp. $319-329$.

[10] - On preservation under homomorphisms and unions of conjunctive queries, Journal of the ACM 53 (2006), 208-237.

[11] G. Ausiello, P. Crescenzi, G. Gambosi, V. Kann, A. Marchetti-Spaccamela, and M. Protasi, Complexity and approximation, Springer-Verlag, Berlin, 1990.

[12] _ Complexity and approximation: Combinatorial optimization problems and their approximability properties., Springer-Verlag, Berlin, 1999.

[13] H.L. Bodlaender, A linear-time algorithm for finding tree-decompositions of small treewidth, SIAM Journal of Computing 25 (1996), no. 6, 1305-1317.

[14] H.L. Bodlaender, J.R. Gilbert, H. Hafsteinsson, and T. Kloks, Approximating treewidth, pathwidth, frontsize, and shortest elimination tree, Journal of Algorithms 18 (1995), 238-255.

[15] C. Borgs, J. Chayes, L. Lovász, V.T. Sós, and K. Vesztergombi, Counting graph homomorphisms, Topics in Discrete Mathematics (M. Klazar, J. Kratochvíl, M. Loebl, J. Matoušek, R. Thomas, and P. Valtr, eds.), Algorithms and Combinatorics, vol. 26, Springer Verlag, 2006, (dedicated to Jarik Nešetřil on the Occasion of his 60 th birthday), pp. 315-371.

[16] O.V. Borodin, On acyclic colorings of planar graphs, Discrete Mathematics 25 (1979), no. 3, 211-236.

[17] A. Brandstädt, H.-O. Le, and J.-M. Vanherpe, Structure and stability number of chair-, co-P- and gem-free graphs revisited, Information Processing Letters 86 (2003), 161-167.

[18] K. Cameron, Induced matchings, Discrete Applied Mathematics 24 (1989), 97-102.

[19] _ Induced matchings in intersection graphs, Discrete Mathematics 278 (2004), 1-9. 
[20] K. Cameron, R. Sritharan, and Y. Tang, Finding a maximum induced matching in weakly chordal graphs, Discrete Mathematics 266 (2003), 133-142.

[21] J.-M. Chang, Induced matchings in asteroidal triple-free graphs, Discrete Applied Mathematics 132 (2004), 67-78.

[22] M. Chrobak and D. Eppstein, Planar orientations with low out-degree and compaction of adjacency matrices, Theoretical Computer Science 86 (1991), 243-266.

[23] F. R. K. Chung, R. L. Graham, and R. M. Wilson, Quasi-random graphs, Combinatorica 9 (1989), no. 4, 345-362.

[24] D. Coppersmith and S. Winograd, Matrix multiplication via arithmetic progressions, J. Symbolic Comput. 9 (1990), 251-280.

[25] B. Courcelle, Graph rewriting: an algebraic and logic approach, Handbook of Theoretical Computer Science (J. van Leeuwen, ed.), vol. 2, Elsevier, Amsterdam, 1990, pp. 142-193.

[26] - The monadic second-order logic of graphs I: recognizable sets of finite graphs, Information Computation 85 (1990), 12-75.

[27] A. Dawar, Finite model theory on tame classes of structures, Mathematical Foundations of Computer Science 2007 (L. Kučera and A. Kučera, eds.), Lecture Notes in Computer Science, vol. 4708, Springer, 2007, pp. 2-12.

[28] _ On preservation theorems in finite model theory, Invited talk at the 6th Panhellenic Logic Symposium - Volos, Greece, July 2007.

[29] A. Dawar, M. Grohe, and S. Kreutzer, Locally excluding a minor, Proc. 22nd IEEE Symp. on Logic in Computer Science, 2007.

[30] J.S. Deogun, T. Kloks, D. Kratsch, and H. Muller, On vertex ranking for permutation and other graphs, Proceedings of the 11th Annual Symposium on Theoretical Aspects of Computer Science (P. Enjalbert, E.W. Mayr, and K.W. Wagner, eds.), Lecture Notes in Computer Science, vol. 775, Springer, 1994, pp. 747-758.

[31] M. DeVos, G. Ding, B. Oporowski, D.P. Sanders, B. Reed, P.D. Seymour, and D. Vertigan, Excluding any graph as a minor allows a low tree-width 2-coloring, Journal of Combinatorial Theory, Series B 91 (2004), 25-41.

[32] R. Diestel, Graph theory, Springer Verlag, 1997.

[33] R. G. Downey and M. R. Fellows, Parameterized computational feasibility, P. Clote, J. Remmel (eds.): Feasible Mathematics II, Boston: Birkhäuser, 1995, pp. 219-244.

[34] _ Parameterized complexity, Springer, 1999.

[35] P. Dreyer, Ch. Malon, and J. Nešetřil, Universal h-colorable graphs without a given configuration, Discrete Math. 250 (2002), 245-25.

[36] W. Duckworth, D.F. Manlove, and M. Zito, On the approximability of the maximum induced matching problem, Journal of Discrete Algorithms 3 (2005), no. 1, 79-91.

[37] W. Duckworth, N.C. Wormald, and M. Zito, Maximum induced matchings of random cubic graphs, Journal of Computational and Applied Mathematics 142 (2002), no. $1,39-50$.

[38] Z. Dvořák, Asymptotical structure of combinatorial objects, Ph.D. thesis, Charles University, Faculty of Mathematics and Physics, 2007.

[39] _ On forbidden subdivision characterization of graph classes, European Journal of Combinatorics (2007), (in press).

[40] H.-D. Ebbinghaus and J. Flum, Finite model theory, Springer-Verlag, 1996. 
[41] G. Elek and B. Szegedy, Limits of hypergraphs, removal and regularity lemmas, a non-standard approach, preprint.

[42] J. Ellis, H. Fan, and M. Fellows, The dominating set problem is fixed parameter tractable for graphs of bounded genus, J. Algorithms 52 (2004), no. 2, 152-168.

[43] D. Eppstein, Subgraph isomorphism in planar graphs and related problems, Proc. 6th Symp. Discrete Algorithms, ACM and SIAM, January 1995, pp. 632-640.

[44] _ Subgraph isomorphism in planar graphs and related problems, Journal of Graph Algorithms \& Applications 3 (1999), no. 3, 1-27.

[45] _ Diameter and treewidth in minor-closed graph families, Algorithmica 27 (2000), 275-291, Special issue on treewidth, graph minors, and algorithms.

[46] P. Erdős, Problems and results in combinatorial analysis and graph theory, Discrete Mathematics 72 (1988), no. 1-3, 81-92.

[47] U. Faigle, U. Kern, H.A. Kierstead, and W.T. Trotter, On the game chromatic number of some classes of graphs, Ars Combinatoria 35 (1993), 143-150.

[48] R.J. Faudree, A. Gyárfás, R.H Schelp, and Zs. Tuza, Induced matchings in bipartite graphs, Discrete Mathematics 78 (1989), no. 1-2, 83-87.

[49] G. Fricke and R. Laskar, Strong matchings in trees, Congressus Numerantium 89 (1992), 239-244

[50] M.R. Garey and D.S. Johnson, Computers and intractability; a guide to the theory of np-completeness, W. H. Freeman \& Co., New York, NY, USA, 1990.

[51] J.R. Gilbert, J.P. Hutchinson, and R.E. Tarjan, A separator theorem for graphs of bounded genus, J. Algorithms (1984), no. 5, 375-390.

[52] M.C. Golumbic and M.C. Laskar, Irredundancy in circular arc graphs, Discrete Applied Mathematics 44 (1993), 79-89.

[53] M.C. Golumbic and M. Lewenstein, New results on induced matchings, Discrete Applied Mathematics 101 (2000), no. 1-3, 157-165.

[54] B. Grünbaum, Acyclic colorings of planar graphs, Israel Journal of Mathematics 14 (1973), 390-408.

[55] D. Guan and X. Zhu, The game chromatic number of outerplanar graphs, Journal of Graph Theory 30 (1999), 67-70.

[56] B. Guenin, Edge coloring plane regular multigraphs, manuscript.

[57] Y. Gurevich, Toward logic tailored for computational complexity, Computation and Proof Theory (M. M. Richter et al., ed.), Lecture Notes in Mathematics, SpringerVerlag, 1984.

[58] R. Häggkvist and P. Hell, Universality of A-mote graphs, Europ. J. Combinatorics 14 (1993), 23-27.

[59] R. Halin, S-functions for graphs, Journal of Geometry 8 (1976), 171-176.

[60] P. Hell and J. Nešetřil, Graphs and homomorphisms, Oxford Lecture Series in Mathematics and its Applications, vol. 28, Oxford University Press, 2004.

[61] W. Hodges, Model theory, Cambridge University Press, 1993.

[62] H.A. Kierstead, personal communication cited in [119].

[63] H.A. Kierstead and D. Yang, Orderings on graphs and game coloring number, Order 20 (2003), 255-264.

[64] D. Kobler and U. Rotics, Finding maximum induced matchings in subclasses of claw-free and $P_{5}$-free graphs, and in graphs with matching and induced matching of equal maximum size, Algorithmica 37 (2003), 327-346. 
[65] Y. Kohayakawa and V. Rödl, Szemerédi's regularity lemma and quasi-randomness, Recent Advances in Algorithmic Combinatorics (B. Reed and C. Linhares-Sales, eds.), CMS Books Math./Ouvrages Math. SMC, vol. 11, Springer, New-York, 2003, pp. $289-347$.

[66] J. Komlós and M. Simonovitz, Szemerédi's regularity lemma and its applications in graph theory, Combinatorics, Paul Erdős is Eighty, vol. 2, János Bolyai Math. Soc., 1993, pp. 295-352.

[67] A.V. Kostochka and L.S. Melnikov, On bounds of the bisection width of cubic graphs, Fourth Czechoslovakian Symposium on Combinatorics, Graphs and Complexity (J. Nesetril and M. Fiedler, eds.), Elsevier, 1992, pp. 151-154.

[68] M. Kreidler and D. Seese, Monadic NP and graph minors, Computer Science Logic, Lecture Notes in Computer Science, vol. 1584, Springer, 1999, pp. 126-141.

[69] B. Larose, C. Loten, and C. Tardif, A characterisation of first-order constraint satisfaction problems, Logical Methods in Computer Science 3 (2007), no. 4, 6, $22 \mathrm{pp}$, (electronic).

[70] L. Libkin, Elements of finite model theory, Springer-Verlag, 2004.

[71] R. Lipton and R.E. Tarjan, A separator theorem for planar graphs, SIAM Journal on Applied Mathematics 36 (1979), no. 2, 177-189.

[72] L. Lovász and B. Szegedy, Szemerédi lemma for the analyst, Geom. Func. Anal. 17 (2007), 252-270.

[73] V.V. Lozin, On maximum induced matchings in bipartite graphs, Information Processing Letters 81 (2002), 7-11.

[74] V.V. Lozin and D. Rautenbach, Some results on graphs without long induced paths, Information Processing Letters 88 (2003), 167-171.

[75] G.L. Miller, S.-H. Teng, W. Thurston, and S.A. Vavasis, Geometric separators for finite-element meshes, SIAM J. on Scientific Computing 19 (1998), no. 2, 364-386.

[76] B.M. Moret, The theory of computation, Addison-Wesley, 1998.

[77] R. Naserasr, Homomorphisms and edge-coloring of planar graphs, Journal of Combinatorial Theory, Series B (2005), to appear.

[78] J. Nešetřil, Aspects of structural combinatorics, Taiwanese J. Math. 3 (1999), no. 4, $381-424$.

[79] J. Nešetřil and P. Ossona de Mendez, Colorings and homomorphisms of minor closed classes, The Goodman-Pollack Festschrift (B. Aronov, S. Basu, J. Pach, and M. Sharir, eds.), Algorithms and Combinatorics, vol. 25, Discrete \& Computational Geometry, 2003, pp. 651-664.

[80] L How many colors may we require?, Prague Midsummer Combinatorial Workshop IX (M. Mareš, ed.), KAM-DIMATIA Series, no. 2004-686, 2004, abstract, pp. 27-30.

[81] _ Cuts and bounds, Discrete Mathematics, Structural Combinatorics - Combinatorial and Computational Aspects of Optimization, Topology and Algebra 302 (2005), no. 1-3, 211-224.

[82] _ Folding, Journal of Combinatorial Theory, Series B 96 (2006), no. 5, 730739.

[83] _ Linear time low tree-width partitions and algorithmic consequences, STOC'06. Proceedings of the 38th Annual ACM Symposium on Theory of Computing, ACM Press, 2006, pp. 391-400. 
[84] _ Tree depth, subgraph coloring and homomorphism bounds, European Journal of Combinatorics 27 (2006), no. 6, 1022-1041.

[85] _ , Fraternal augmentations, arrangeability and linearly bounded Ramsey numbers, SIAM Journal on Discrete Mathematics (2007), submitted.

[86] _ Induced matchings and induced paths in graphs, Tech. Report 2007-810, KAM-DIMATIA Series, 2007.

[87] _ First order properties on nowhere dense structures, Journal of Symbolic Logic (2008), submitted.

[88] _ Grad and classes with bounded expansion I. decompositions, European Journal of Combinatorics 29 (2008), no. 3, 760-776.

[89] Grad and classes with bounded expansion II. algorithmic aspects, European Journal of Combinatorics 29 (2008), no. 3, 777-791.

[90] — Grad and classes with bounded expansion III. restricted graph homomorphism dualities, European Journal of Combinatorics (2008), (in press).

[91] _ On nowhere dense graphs, European Journal of Combinatorics (2008), submitted.

[92] J. Nešetrril and S. Poljak, Complexity of the subgraph problem, Comment. Math. Univ. Carol. 26.2 (1985), 415-420.

[93] J. Nešetřil and A. Pultr, On classes of relations and graphs determined by subobjects and factorobjects, Discrete Mathematics 22 (1978), 287-300.

[94] J. Nešetřil and R. Śámal, Tension continuous maps-their structure and applications, Tech. Report 2005-242, ITI Series, 2005, (to appear in European J. Comb.).

[95] J. Nešetřil and S. Shelah, Order of countable graphs, European J. Comb. 24 (2003), 649-663.

[96] J. Nešetřil and I. Švejdarová, Diameter of duals are linear, Tech. Report 2005-729, KAM-DIMATIA Series, 2005.

[97] J. Nešetřil and C. Tardif, Duality theorems for finite structures (characterizing gaps and good characterizations), Journal of Combinatorial Theory, Series B 80 (2000), 80-97.

[98] _ Short answers to exponentially long questions: Extremal aspects of homomorphism duality, SIAM Journal of Discrete Mathematics 19 (2005), no. 4, 914-920.

[99] C. H. Papadimitriou and M. Yannakakis, Optimization, approximation and complexity classes, Journal of Computer and System Sciences 43 (1991), 425-440.

[100] S. Plotkin, S. Rao, and W.D. Smith, Shallow excluded minors and improved graph decomposition, 5th Symp. Discrete Algorithms, SIAM, 1994.

[101] N. Robertson and P.D. Seymour, Graph minors. I. Excluding a forest, Journal of Combinatorial Theory, Series B 35 (1983), 39-61.

[102] _ Graph minors - a survey, Surveys in Combinatorics (I. Anderson, ed.), Cambridge University Press, 1985, pp. 153-171.

[103] Graph Minors. II. Algorithmic aspects of tree-width, Journal of Algorithms 7 (1986), 309-322.

[104] _ Graph Minors. VIII., Journal of Combinatorial Theory, Series B 48 (1990), no. $2,227-254$.

[105] B. Rossman, Homomorphisms and first-order logic, Journal of the ACM (2007), submitted. 
[106] P. Schaffer, Optimal node ranking of trees in linear time, Information Processing Letters 33 (1989/90), 91-96.

[107] A. Steger and M.-l. Yu, On induced matchings, Discrete Mathematics 120 (1993), no. 1-3, 291-295.

[108] L.J. Stockmeyer and V.V. Vazirani, NP-completeness of some generalizations of the maximum matching problem, Information Processing Letters 15 (1982), no. 1, $14-19$.

[109] A. Stolboushkin, Finite monotone properties, Proc. 10th IEEE Symp. on Logic in Computer Science, 1995, pp. 324-330.

[110] L. Sunil Chandran, A high girth graph construction, SIAM J. Discret. Math. 16 (2003), no. 3, 366-370.

[111] E. Szemerédi, Regular partitions of graphs, Problémes combinatoires et théorie des graphes, CNRS, 1976, pp. 399-401.

[112] W. Tait, A counterexample to a conjecture of Scott and Suppes, Journal of Symbolic Logic 24 (1959), 15-16.

[113] T. Tao, The dichotomy between structure and randomness, arithmetic progression, and the primes, Proceedings of the Intersnational Congress of Mathematicians (Madrid 2006) (European Math. Society, ed.), vol. 1, 2007, pp. 581-608.

[114] S.-H. Teng, Combinatorial aspects of geometric graphs, Computational Geometry (1998), no. 9, 277-287.

[115] C. Thomassen, A short list color proof of Grötzsch theorem, Journal of Combinatorial Theory, Series B 88 (2003), 189-192.

[116] K. Wagner, Über eine Eigenschaft der Ebenen Komplexe, Mathematische Annalen 114 (1937), 570-590.

[117] R. Yuster, Combinatorial and computational aspects of graph packing and graph decompositions, Computer Science Review 1 (2007), no. 1, 12-26.

[118] X. Zhu, The game coloring number of planar graphs, Journal of Combinatorial Theory, Series B 75 (1999), no. 2, 245-258.

[119] , The game coloring number of pseudo partial $k$-trees, Discrete Mathematics 215 (2000), 245-262.

[120] C Colouring graphs with bounded generalized colouring number, Discrete Mathematics (2006), submitted.

[121] , Refined activation strategy for the marking game, Journal of Combinatorial Theory, Series B (2007), in press.

[122] M. Zito, Maximum induced matchings in regular graphs and trees, Proceedings of WG '99: the 25th International Workshop on Graph-Theoretic Concepts in Computer Science, Lecture Notes in Computer Science, vol. 1665, 1999, pp. 89100 .

Department of Applied Mathematics and Institute of Theoretical Computer Science (ITI), Charles University, Malostranské nám.25, 11800 Praha 1, Czech REPUBLIC

E-mail address: nesetril@kam.ms.mff.cuni.cz

Centre d'Analyse et de Mathématiques Sociales, CNRS, UMR 8557, 54 Bd RASPAIL, 75006 PARIS, FRANCE

E-mail address: pom@ehess.fr 